\title{
Complex scalar dark matter in the gauged two-Higgs-doublet model
}

\author{
Chuan-Ren Chen, ${ }^{1}$ Yu-Xiang Lin $\odot,{ }^{1}$ Chrisna Setyo Nugroho $\odot,{ }^{2,3}$ Raymundo Ramos $\odot,{ }^{2}$ \\ Yue-Lin Sming Tsai, ${ }^{2,4}$ and Tzu-Chiang Yuan ${ }^{2}$ \\ ${ }^{1}$ Department of Physics, National Taiwan Normal University, Taipei 116, Taiwan \\ ${ }^{2}$ Institute of Physics, Academia Sinica, Nangang, Taipei 11529, Taiwan \\ ${ }^{3}$ Physics Division, National Center for Theoretical Sciences, Hsinchu 300, Taiwan \\ ${ }^{4}$ Key Laboratory of Dark Matter and Space Astronomy, Purple Mountain Observatory, \\ Chinese Academy of Sciences, Nanjing 210008, China
}

(Received 23 December 2019; accepted 30 January 2020; published 27 February 2020)

\begin{abstract}
The complex scalar dark matter (DM) candidate in the gauged two-Higgs-doublet model, stabilized by a peculiar hidden parity ( $h$ parity), is studied in detail. We explore the parameter space for the DM candidate by taking into account the most recent DM constraints from various experiments, in particular, the PLANCK relic density measurement and the current DM direct detection limit from XENON1T. We separate our analysis in three possible compositions for the mixing of the complex scalar. We first constrain our parameter space with the vacuum stability and perturbative unitarity conditions for the scalar potential, LHC Higgs measurements, plus Drell-Yan and electroweak precision test constraints on the gauge sector. We find that DM dominated by composition of the inert doublet scalar is completely excluded by further combining the previous constraints with both the latest results from PLANCK and XENON1T. We also demonstrate that the remaining parameter space with two other DM compositions can be further tested by indirect detection like the future Cherenkov Telescope Array gamma-ray telescope.
\end{abstract}

DOI: 10.1103/PhysRevD.101.035037

\section{INTRODUCTION}

Dark matter (DM) has become one of the most discussed topics in cosmology, astrophysics, and particle physics. However, besides the indirect evidence from the power spectrum from the cosmic microwave background radiation and the galaxy rotational curves which provide strong hints for the need of DM, current experiments for DM direct detection, indirect detection, and collider searches still show no clue for the nature of DM. Currently, the best description of the early history of the Universe is given by the $\Lambda \mathrm{CDM}$ model which assumes the presence of dark energy and cold DM in additional to the ordinary matter. The leading hypothesis is that this cold DM is composed of weakly interacting massive particle (WIMP) that was thermally produced just like the other Standard Model (SM) particles in the early Universe. It is well known that the most compelling feature of WIMP DM is that after freeze-out whence the DM reaction rates fall behind the Hubble expansion rate of the Universe, it is possible to achieve the correct relic abundance with an electroweak

Published by the American Physical Society under the terms of the Creative Commons Attribution 4.0 International license. Further distribution of this work must maintain attribution to the author(s) and the published article's title, journal citation, and DOI. Funded by SCOAP . sized annihilation cross section with a WIMP mass of a few hundred $\mathrm{GeV}$ to a few $\mathrm{TeV}$.

On the collider phenomenology side, we now know that all the major decay modes of the SM $125 \mathrm{GeV}$ Higgs discovered on July 4, 2012 at the Large Hadron Collider (LHC) have been observed except the $Z \gamma$ and $\mu^{+} \mu^{-}$modes. So far, all the experimental results agree with the SM predictions within 10\%-15\%. Nevertheless, there are still some rooms for new physics. A particular class of models that extends simply the scalar sector of the SM to address new physics is quite popular. The most well-known example is the general two-Higgs-doublet model (2HDM), which has several variants and resulted in very rich phenomenology. For a review of 2HDM and its phenomenology, see, e.g., $[1,2]$. One of the interesting variants of $2 \mathrm{HDM}$ is to impose a $\mathcal{Z}_{2}$ symmetry in the model so that the second Higgs doublet is $\mathcal{Z}_{2}$ odd and then can be a DM candidate. This is the inert Higgs doublet model (IHDM) [3], and many detailed phenomenological studies [4-27] have been performed over the years. Furthermore, the idea that this $\mathcal{Z}_{2}$ symmetry emerges accidentally in a renormalizable gauged twoHiggs-doublet model (G2HDM) has been explored recently in [28]. In G2HDM, the two Higgs doublets are grouped together in an irreducible doublet representation of an extra non-Abelian $S U(2)_{H}$ gauge group. Besides the new hidden $S U(2)_{H}$, the SM gauge group is extended by including a new $U(1)_{X}$ symmetry. The scalar sector is further extended by 
including a new $S U(2)_{H}$ doublet and a triplet, both singlets under the SM gauge group.

Although any electrically neutral $\mathcal{Z}_{2}$-odd particle in G2HDM can be considered a DM candidate, such as $W^{\prime}$ or heavy new neutrinos, in this work we choose to concentrate on the phenomenology of the complex scalar DM candidate. The reason is mainly that we want to present this model as a viable alternative to IHDM and as such our setup is focused in providing a light, neutral, and $\mathcal{Z}_{2}$-odd complex scalar. Another reason is practicality, given that our setup is complicated enough to distinguish at least three main types of DM candidates coming only from the scalar sector due to mixing effects. We will study in detail the differences, similarities, and results of these three possibilities. Phenomenologically, we expect all of them to communicate with the SM through Higgs portal type interactions $[29,30]$. However, we will demonstrate that the SM $Z$ boson as well as its heavier siblings in G2HDM will also play non-negligible roles as mediators in various DM processes in relic density, direct and indirect detection, especially for the inert doubletlike DM case.

In the past, some collider phenomenology of G2HDM has been studied [31-33]. It was determined that Drell-Yan type signals may help detect the G2HDM $Z^{\prime}$ in the high luminosity upgrade of the LHC [32] and that enhancement of pair production of Higgs boson in the LHC is moderate compared to the SM [33]. In a recent study, it has been determined that G2HDM has a viable scalar sector parameter space [34], compatible with vacuum stability and perturbative unitarity conditions, as well as Higgs phenomenology constraints from the LHC. The gauge sector is constrained by electroweak precision tests (EWPT) [35], setting limits on the masses of the new gauge bosons and the gauge sector parameter space. It is precisely these two recent studies on the scalar and gauge sectors constraints (SGSC) that we will take as starting point for our study, thus ensuring that the final constrained parameter space is consistent with previous studies and that our result has a stronger relevance.

This paper is organized as follows. In Sec. II, we briefly recall some salient features of the G2HDM model, in particular the scalar potential and mass spectra. In Sec. III, we point out after spontaneous symmetry breaking there exists an accidental discrete $\mathcal{Z}_{2}$ symmetry in the whole Lagrangian of G2HDM. We classify all the particles in the model according to whether they are even or odd under this discrete symmetry, dubbed as $h$ parity. This residual symmetry forbids the lightest particle in the hidden sector to decay and hence it, if electrically neutral, may be a cold DM candidate in the model. We discuss further the compositions of complex scalar dark matter that is relevant in this work. In Sec. IV, we discuss the DM constraints included in our analysis and how they are affected by our setup in more general terms. We describe how relic density (RD), direct detection (DD), and indirect detection (ID)
TABLE I. Matter contents and their quantum number assignments in G2HDM. Note that $H$ here is written explicitly as an $S U(2)_{H}$ doublet and, thus, the T stands for $S U(2)_{H}$ transposition. For doublets of a single $S U(2)$, T stands for transposition under that same $S U(2)$.

\begin{tabular}{lc}
\hline \hline Matter fields & $S U(3)_{C} \times S U(2)_{L} \times S U(2)_{H} \times U(1)_{Y} \times U(1)_{X}$ \\
\hline$H=\left(H_{1}, H_{2}\right)^{\mathrm{T}}$ & $(1,2,2,1 / 2,1)$ \\
$\Delta_{H}$ & $(1,1,3,0,0)$ \\
$\Phi_{H}$ & $(1,1,2,0,1)$ \\
$Q_{L}=\left(u_{L}, d_{L}\right)^{\mathrm{T}}$ & $(3,2,1,1 / 6,0)$ \\
$U_{R}=\left(u_{R}, u_{R}^{H}\right)^{\mathrm{T}}$ & $(3,1,2,2 / 3,1)$ \\
$D_{R}=\left(d_{R}^{H}, d_{R}\right)^{\mathrm{T}}$ & $(3,1,2,-1 / 3,-1)$ \\
$L_{L}=\left(\nu_{L}, e_{L}\right)^{\mathrm{T}}$ & $(1,2,1,-1 / 2,0)$ \\
$N_{R}=\left(\nu_{R}, \nu_{R}^{H}\right)^{\mathrm{T}}$ & $(1,1,2,0,1)$ \\
$E_{R}=\left(e_{R}^{H}, e_{R}\right)^{\mathrm{T}}$ & $(1,1,2,-1,-1)$ \\
$\nu_{L}^{H}$ & $(1,1,1,0,0)$ \\
$e_{L}^{H}$ & $(1,1,1,-1,0)$ \\
$u_{L}^{H}$ & $(3,1,1,2 / 3,0)$ \\
$d_{L}^{H}$ & \\
\hline \hline
\end{tabular}

measurements constrain each of the three different compositions of complex scalar DM considered. We also discuss the collider searches of DM from the monojet plus missing energy search and invisible Higgs decay. In Sec. V, after a brief description of the methodology used in our numerical analysis, we present the results of our analysis. Finally, in Sec. VI, we summarize our findings and conclude, including a brief comment on future detectability. Some Feynman rules that are most relevant to the processes discussed in this work are collected in the Appendix.

\section{THE G2HDM MODEL}

\section{A. Matter content}

The gauge symmetry group of G2HDM expands the SM gauge group $S U(2)_{L} \times U(1)_{Y}$ by adding a hidden sector of $S U(2)_{H} \times U(1)_{X}$. In the scalar sector, we have the two $S U(2)_{L}$ Higgs doublets $H_{1}$ and $H_{2}$ both paired into an $S U(2)_{H}$ doublet $H$. The two scalar SM singlets, $\Delta_{H}$ and $\Phi_{H}$, have been put into the triplet and doublet representations of $S U(2)_{H}$, respectively. In order to construct gauge invariant Yukawa couplings, new right-handed heavy fermions have been added as $S U(2)_{H}$ companions of the SM right-handed fermions, pairing both of them together into $S U(2)_{H}$ doublets, but remaining $S U(2)_{L}$ singlets. Anomaly cancellation requires further that we add two pairs of left-handed heavy leptons and two pairs of lefthanded heavy quarks for each family, all of them are singlets under both $S U(2)$ groups and under $U(1)_{X}$. We note that the $S U(2)_{H}$ is not the same as the $S U(2)_{R}$ in leftright symmetric models $[36,37]$. The $W^{\prime(p, m)}$ in G2HDM does not carry electric charge, while the $W^{\prime \pm}$ in left-right 
symmetric models does. Thus, we use the superscripts $p$ and $m$ to label them, instead of + and - . We also note that nonsterile right-handed neutrinos $\nu_{l R} \mathrm{~S}$ introduced in the mirror fermion models of electroweak scale right-handed neutrinos [38-40] are in a different manner. In the mirror fermion models, $\nu_{l R} \mathrm{~S}$ are grouped with mirror charged leptons $l_{R}^{M}$ s to form $S U(2)_{L}$ doublets. Here in G2HDM, they are grouped with new heavy right-handed neutrinos $\nu_{l R}^{H}$ to form $S U(2)_{H}$ doublets instead. For other related ideas extending the $2 \mathrm{HDM}$ with extra gauge symmetries to address flavor problem, dark matter, and neutrino masses, see, e.g., [41-46]. The matter contents of the G2HDM model and their respective quantum numbers are listed in Table I.

\section{B. Scalar potential and constraints}

\section{Scalar potential}

For this work, we will be using the scalar potential from Ref. [34] that extends the original potential of Ref. [28] by adding two new terms with couplings $\lambda_{H}^{\prime}$ and $\lambda_{H \Phi}^{\prime}$. The most general scalar potential that respects the G2HDM symmetries can be divided into four different parts,

$V_{T}=V(H)+V\left(\Phi_{H}\right)+V\left(\Delta_{H}\right)+V_{\text {mix }}\left(H, \Delta_{H}, \Phi_{H}\right)$.

The first term $V(H)$ in Eq. (1) consists of the two Higgs doublets $H_{1}$ and $H_{2}$ only and is given by

$$
\begin{aligned}
V(H)= & \mu_{H}^{2}\left(H^{\alpha i} H_{\alpha i}\right)+\lambda_{H}\left(H^{\alpha i} H_{\alpha i}\right)^{2} \\
& +\frac{1}{2} \lambda_{H}^{\prime} \epsilon_{\alpha \beta} \epsilon^{\gamma \delta}\left(H^{\alpha i} H_{\gamma i}\right)\left(H^{\beta j} H_{\delta j}\right) \\
= & \mu_{H}^{2}\left(H_{1}^{\dagger} H_{1}+H_{2}^{\dagger} H_{2}\right)+\lambda_{H}\left(H_{1}^{\dagger} H_{1}+H_{2}^{\dagger} H_{2}\right)^{2} \\
& +\lambda_{H}^{\prime}\left(-H_{1}^{\dagger} H_{1} H_{2}^{\dagger} H_{2}+H_{1}^{\dagger} H_{2} H_{2}^{\dagger} H_{1}\right),
\end{aligned}
$$

where Greek and Latin letters refer to $S U(2)_{H}$ and $S U(2)_{L}$ indices, respectively, both of which run from 1 to 2 , and we use the notation $H^{\alpha i}=H_{\alpha i}^{*}$. From the second line of Eq. (2), one can clearly see that $V(H)$ has the discrete $\mathcal{Z}_{2}$ symmetry of $H_{1} \rightarrow H_{1}$ and $H_{2} \rightarrow-H_{2}$. Since $V(H)$ contains all the renormalizable terms constructed solely from $H_{1}$ and $H_{2}$, this discrete symmetry is automatically present. Recall that in general 2HDM, one needs to impose this discrete symmetry to avoid unwanted terms that may lead to flavor changing neutral current in the HiggsYukawa interactions at the tree level. The second term $V\left(\Phi_{H}\right)$ is for the $S U(2)_{H}$ doublet $\Phi_{H}$ only and given by

$$
\begin{aligned}
V\left(\Phi_{H}\right) & =\mu_{\Phi}^{2} \Phi_{H}^{\dagger} \Phi_{H}+\lambda_{\Phi}\left(\Phi_{H}^{\dagger} \Phi_{H}\right)^{2} \\
& =\mu_{\Phi}^{2}\left(\Phi_{1}^{*} \Phi_{1}+\Phi_{2}^{*} \Phi_{2}\right)+\lambda_{\Phi}\left(\Phi_{1}^{*} \Phi_{1}+\Phi_{2}^{*} \Phi_{2}\right)^{2},
\end{aligned}
$$

where $\Phi_{H}=\left(\Phi_{1}, \Phi_{2}\right)^{\mathrm{T}}$. The third term is for the $S U(2)_{H}$ triplet $\Delta_{H}$ and is given by

$$
\begin{aligned}
V\left(\Delta_{H}\right) & =-\mu_{\Delta}^{2} \operatorname{Tr}\left(\Delta_{H}^{2}\right)+\lambda_{\Delta}\left(\operatorname{Tr}\left(\Delta_{H}^{2}\right)\right)^{2} \\
& =-\mu_{\Delta}^{2}\left(\frac{1}{2} \Delta_{3}^{2}+\Delta_{p} \Delta_{m}\right)+\lambda_{\Delta}\left(\frac{1}{2} \Delta_{3}^{2}+\Delta_{p} \Delta_{m}\right)^{2},
\end{aligned}
$$

where

$$
\begin{aligned}
& \Delta_{H}=\left(\begin{array}{cc}
\Delta_{3} / 2 & \Delta_{p} / \sqrt{2} \\
\Delta_{m} / \sqrt{2} & -\Delta_{3} / 2
\end{array}\right)=\Delta_{H}^{\dagger} \text { with } \\
& \Delta_{m}=\left(\Delta_{p}\right)^{*} \text { and }\left(\Delta_{3}\right)^{*}=\Delta_{3} .
\end{aligned}
$$

Furthermore, unlike other models with $S U(2)_{L}$ triplet Higgs, the off-diagonal components $\Delta_{p, m}$ do not carry electric charge. We use the subscripts $p$ and $m$ to label them instead of + and - , in the same way as the new gauge bosons $W^{\prime(p, m)}$. Finally, the last term $V_{\text {mix }}$ consists all three scalars $H, \Phi_{H}$, and $\Delta_{H}$,

$$
\begin{aligned}
V_{\text {mix }} & \left(H, \Delta_{H}, \Phi_{H}\right) \\
= & +M_{H \Delta}\left(H^{\dagger} \Delta_{H} H\right)-M_{\Phi \Delta}\left(\Phi_{H}^{\dagger} \Delta_{H} \Phi_{H}\right) \\
& +\lambda_{H \Phi}\left(H^{\dagger} H\right)\left(\Phi_{H}^{\dagger} \Phi_{H}\right)+\lambda_{H \Phi}^{\prime}\left(H^{\dagger} \Phi_{H}\right)\left(\Phi_{H}^{\dagger} H\right) \\
& +\lambda_{H \Delta}\left(H^{\dagger} H\right) \operatorname{Tr}\left(\Delta_{H}^{2}\right)+\lambda_{\Phi \Delta}\left(\Phi_{H}^{\dagger} \Phi_{H}\right) \operatorname{Tr}\left(\Delta_{H}^{2}\right) .
\end{aligned}
$$

Equation (6) can be expanded further in terms of the component fields of $H, \Delta_{H}$, and $\Phi_{H}$ as follows:

$$
\begin{aligned}
V_{\text {mix }}\left(H, \Delta_{H}, \Phi_{H}\right)= & +M_{H \Delta}\left(\frac{1}{\sqrt{2}} H_{1}^{\dagger} H_{2} \Delta_{p}+\frac{1}{2} H_{1}^{\dagger} H_{1} \Delta_{3}+\frac{1}{\sqrt{2}} H_{2}^{\dagger} H_{1} \Delta_{m}-\frac{1}{2} H_{2}^{\dagger} H_{2} \Delta_{3}\right) \\
& -M_{\Phi \Delta}\left(\frac{1}{\sqrt{2}} \Phi_{1}^{*} \Phi_{2} \Delta_{p}+\frac{1}{2} \Phi_{1}^{*} \Phi_{1} \Delta_{3}+\frac{1}{\sqrt{2}} \Phi_{2}^{*} \Phi_{1} \Delta_{m}-\frac{1}{2} \Phi_{2}^{*} \Phi_{2} \Delta_{3}\right) \\
& +\lambda_{H \Phi}\left(H_{1}^{\dagger} H_{1}+H_{2}^{\dagger} H_{2}\right)\left(\Phi_{1}^{*} \Phi_{1}+\Phi_{2}^{*} \Phi_{2}\right)+\lambda_{H \Phi}^{\prime}\left(H_{1}^{\dagger} H_{1} \Phi_{1}^{*} \Phi_{1}+H_{2}^{\dagger} H_{2} \Phi_{2}^{*} \Phi_{2}+H_{1}^{\dagger} H_{2} \Phi_{2}^{*} \Phi_{1}+H_{2}^{\dagger} H_{1} \Phi_{1}^{*} \Phi_{2}\right) \\
& +\lambda_{H \Delta}\left(H_{1}^{\dagger} H_{1}+H_{2}^{\dagger} H_{2}\right)\left(\frac{1}{2} \Delta_{3}^{2}+\Delta_{p} \Delta_{m}\right)+\lambda_{\Phi \Delta}\left(\Phi_{1}^{*} \Phi_{1}+\Phi_{2}^{*} \Phi_{2}\right)\left(\frac{1}{2} \Delta_{3}^{2}+\Delta_{p} \Delta_{m}\right)
\end{aligned}
$$


Interestingly, the whole scalar potential $V_{T}$ in Eq. (1) is invariant under the combined discrete symmetry $H_{1} \rightarrow H_{1}$, $H_{2} \rightarrow-H_{2}, \Phi_{1} \rightarrow-\Phi_{1}, \Phi_{2} \rightarrow \Phi_{2}, \Delta_{3} \rightarrow \Delta_{3}$, and $\Delta_{p, m} \rightarrow$ $-\Delta_{p, m}$. Since $V_{T}$ consists of all renormalization terms constructed out of $H, \Delta_{H}$, and $\Phi_{H}$, this discrete symmetry can be viewed as an accidental one in the scalar sector of the model. In fact, one can extend this discrete symmetry to the whole renormalizable Lagrangian of G2HDM. We will discuss this further in Sec. III.

\section{Spontaneous symmetry breaking}

The gauge symmetry of G2HDM is broken spontaneously by the vacuum expectation values (VEVs) of $\left\langle H_{1}\right\rangle=(0, v / \sqrt{2})^{\mathrm{T}},\left\langle\Phi_{2}\right\rangle=v_{\Phi} / \sqrt{2}$, and $\left\langle\Delta_{3}\right\rangle=-v_{\Delta}$. In Ref. [28], we demonstrated that $v_{\Delta}$ satisfies a cubic equation with all coefficients expressed in terms of the fundamental parameters in the scalar potential. Solutions of $v_{\Delta}$ can be found either analytically or numerically and plug into the linear coupled equations for $v^{2}$ and $v_{\Phi}^{2}$ that can then be solved straightforwardly. Since $v_{\Delta}$ has three different roots in general, the correct one will be picked by minimum energy requirement. Thus, the symmetry breaking of $S U(2)_{L} \times U(1)_{Y} \times U(1)_{X}$ in G2HDM is induced or triggered by the triplet VEV $v_{\Delta}$ which breaks $S U(2)_{H}$. Note that the sign of $\mu_{\Delta}^{2}$ is negative with respect to $\mu_{\Phi}^{2}$ and $\mu_{H}^{2}$. If $\mu_{\Delta}^{2}>0, S U(2)_{H}$ is spontaneously broken by the VEV $v_{\Delta} \neq 0$. After $S U(2)_{H}$ symmetry breaking is triggered, the vacuum alignment of $\Phi_{H}$ is controlled by the quadratic terms for $\Phi_{1}$ and $\Phi_{2}$ given by

$$
\begin{gathered}
\mu_{\Phi}^{2}+\frac{1}{2} M_{\Phi \Delta} v_{\Delta}+\frac{1}{2} \lambda_{\Phi \Delta} v_{\Delta}^{2}+\frac{1}{2}\left(\lambda_{H \Phi}+\lambda_{H \Phi}^{\prime}\right) v^{2}, \\
\mu_{\Phi}^{2}-\frac{1}{2} M_{\Phi \Delta} v_{\Delta}+\frac{1}{2} \lambda_{\Phi \Delta} v_{\Delta}^{2}+\frac{1}{2} \lambda_{H \Phi} v^{2},
\end{gathered}
$$

respectively. The parameters $M_{\Phi \Delta}, \lambda_{\Phi \Delta}, \lambda_{H \Phi}$, and $\lambda_{H \Phi}^{\prime}$ can be either positive or negative independent of the sign of $\mu_{\Phi}^{2}$, meaning that Eqs. (8) and (9) can be positive and negative, respectively. One can achieve $\left\langle\Phi_{1}\right\rangle=0$ and $\left\langle\Phi_{2}\right\rangle \neq 0$ by making judicious choices of the parameters. Furthermore, $S U(2)_{L}$ symmetry breaking is controlled by the quadratic terms of $H_{1}$ and $H_{2}$. After expanding the potential, the coefficients for them are

$$
\begin{gathered}
\mu_{H}^{2}-\frac{1}{2} M_{H \Delta} v_{\Delta}+\frac{1}{2} \lambda_{H \Delta} v_{\Delta}^{2}+\frac{1}{2} \lambda_{H \Phi} v_{\Phi}^{2}, \\
\mu_{H}^{2}+\frac{1}{2} M_{H \Delta} v_{\Delta}+\frac{1}{2} \lambda_{H \Delta} v_{\Delta}^{2}+\frac{1}{2}\left(\lambda_{H \Phi}+\lambda_{H \Phi}^{\prime}\right) v_{\Phi}^{2},
\end{gathered}
$$

respectively. Similar to the $\Phi_{H}$ case, the parameters involved can be either positive or negative independently of the sign of $\mu_{H}^{2}$, but this time Eqs. (10) and (11) would be negative and positive, respectively. In this case, the gauge symmetry $S U(2)_{L}$ is broken by the vacuum alignment $\left\langle H_{1}\right\rangle \neq 0$ and $\left\langle H_{2}\right\rangle=0$. In our numerical scan for the parameter space in Sec. V, we will search for parameters such that Eqs. (8) and (11) are positive while Eqs. (9) and (10) are negative in order to achieve the desired vacuum alignment. The $\mu_{H}^{2}, \mu_{\Phi}^{2}$, and $\mu_{\Delta}^{2}$ parameters will be fixed using the VEV equations (Eqs. (18)-(20) of Ref. [28]). Since $H_{1}, \Phi_{2}$, and $\Delta_{3}$ are all even under $\mathcal{Z}_{2}$, the discrete symmetry is not broken by their VEVs. Therefore, the $\mathcal{Z}_{2^{-}}$ odd $\mathrm{H}_{2}$ can become a DM candidate as long as it is lighter than all other $\mathcal{Z}_{2}$-odd particles in the model.

\section{Theoretical and phenomenological constraints on the scalar potential}

We will begin our analysis by considering the conditions determined in Ref. [34] for the scalar sector parameter space. Namely, we want to start with a parameter space that leaves the scalar potential bounded from below, with couplings that remain within perturbative unitarity ranges and make sure that we can actually achieve a sufficiently SM-like Higgs with a $\sim 125 \mathrm{GeV}$ mass and that can pass the limits set by the LHC.

While for the minimum of the potential one checks the quadratic terms, to ensure that the scalar potential is bounded from below for large-field values one is mainly concerned with the quartic terms. In Ref. [34], it was shown that copositivity criteria [47-49] is enough to find conditions for the potential to be bounded from below and have a stable vacuum. The copositive conditions are

$$
\begin{gathered}
\tilde{\lambda}_{H}(\eta) \geq 0, \quad \lambda_{\Phi} \geq 0, \quad \lambda_{\Delta} \geq 0, \\
\Lambda_{H \Phi}(\xi, \eta) \equiv \tilde{\lambda}_{H \Phi}(\xi)+2 \sqrt{\tilde{\lambda}_{H}(\eta) \lambda_{\Phi}} \geq 0, \\
\Lambda_{H \Delta}(\eta) \equiv \lambda_{H \Delta}+2 \sqrt{\tilde{\lambda}_{H}(\eta) \lambda_{\Delta}} \geq 0, \\
\Lambda_{\Phi \Delta} \equiv \lambda_{\Phi \Delta}+2 \sqrt{\lambda_{\Phi} \lambda_{\Delta}} \geq 0 \\
\Lambda_{H \Phi \Delta}(\xi, \eta) \equiv \sqrt{\tilde{\lambda}_{H}(\eta) \lambda_{\Phi} \lambda_{\Delta}}+\frac{1}{2}\left(\tilde{\lambda}_{H \Phi}(\xi) \sqrt{\lambda_{\Delta}}\right. \\
\left.+\lambda_{H \Delta} \sqrt{\lambda_{\Phi}}+\lambda_{\Phi \Delta} \sqrt{\tilde{\lambda}_{H}(\eta)}\right) \\
+\frac{1}{2} \sqrt{\Lambda_{H \Phi}(\xi, \eta) \Lambda_{H \Delta}(\eta) \Lambda_{\Phi \Delta}} \geq 0
\end{gathered}
$$

where the parameters $\xi$ and $\eta$ can have any value in the ranges $0 \leq \xi \leq 1$ and $-1 / 4 \leq \eta \leq 0$.

On the other hand, we have to make sure that our parameter space remains within perturbative limits. Again, we look only at quartic couplings since $2 \rightarrow 2$ scattering processes induced by cubic couplings are suppressed by their propagators while quartic couplings are not. After checking all the possible $2 \rightarrow 2$ scattering processes, the final ranges allowed by perturbative unitarity are 


$$
\begin{aligned}
\left|\lambda_{i}\left(\mathcal{M}_{1}\right)\right| & \leq 8 \pi, \quad \forall i=(1, \ldots, 10), \\
\left|\lambda_{H}\right| & \leq 4 \pi,\left|\lambda_{H}^{\prime}\right| \leq 8 \sqrt{2} \pi,\left|2 \lambda_{H} \pm \lambda_{H}^{\prime}\right| \leq 8 \pi,\left|\lambda_{\Phi}\right| \leq 4 \pi,\left|\lambda_{\Delta}\right| \leq 4 \pi, \\
\left|\lambda_{H \Phi}\right| & \leq 8 \pi,\left|\tilde{\lambda}_{H \Phi}\right|=\left|\lambda_{H \Phi}+\lambda_{H \Phi}^{\prime}\right| \leq 8 \pi,\left|\lambda_{H \Phi}^{\prime}\right| \leq 8 \sqrt{2} \pi, \\
\left|\lambda_{H \Delta}\right| & \leq 8 \pi,\left|\lambda_{\Phi \Delta}\right| \leq 8 \pi,
\end{aligned}
$$

where

$$
\begin{aligned}
\lambda_{1} & =2 \lambda_{H}, \quad \lambda_{2}=2 \lambda_{\Phi}, \quad \lambda_{3}=2 \lambda_{\Delta}, \quad \lambda_{4,5}=2 \lambda_{H} \pm \lambda_{H}^{\prime}, \\
\lambda_{6,7} & =\tilde{\lambda}_{H}^{+}+\lambda_{\Phi} \pm \sqrt{2 \lambda_{H \Phi}^{\prime 2}+\left(\tilde{\lambda}_{H}^{+}-\lambda_{\Phi}\right)^{2}},
\end{aligned}
$$

with $\tilde{\lambda}_{H}^{+} \equiv \lambda_{H}+\lambda_{H}^{\prime} / 2$ and $\lambda_{8,9,10}$ given by the three roots of the equation $\lambda^{3}+a \lambda^{2}+b \lambda+c=0$ with

$$
\begin{aligned}
& a=-5 \lambda_{\Delta}-6 \lambda_{\Phi}-10 \lambda_{H}+\lambda_{H}^{\prime}, \\
& b=-6 \lambda_{H \Delta}^{2}-3 \lambda_{\Phi \Delta}^{2}+5 \lambda_{\Delta}\left(10 \lambda_{H}-\lambda_{H}^{\prime}+6 \lambda_{\Phi}\right)+6 \lambda_{\Phi}\left(10 \lambda_{H}-\lambda_{H}^{\prime}\right)-8\left(\lambda_{H \Phi}+\lambda_{H \Phi}^{\prime} / 2\right)^{2}, \\
& c=36 \lambda_{\Phi} \lambda_{H \Delta}^{2}-24 \lambda_{H \Delta} \lambda_{\Phi \Delta}\left(\lambda_{H \Phi}+\lambda_{H \Phi}^{\prime} / 2\right)+40 \lambda_{\Delta}\left(\lambda_{H \Phi}+\lambda_{H \Phi}^{\prime} / 2\right)^{2}+\left(3 \lambda_{\Phi \Delta}^{2}-30 \lambda_{\Delta} \lambda_{\Phi}\right)\left(10 \lambda_{H}-\lambda_{H}^{\prime}\right) .
\end{aligned}
$$

On the phenomenological side, we will require the presence of a SM Higgs with a mass of $125.09 \pm 0.24 \mathrm{GeV}$ and a signal strength for the Higgs decay into two photos of $\mu_{g g H}^{\gamma \gamma}=0.81_{-0.18}^{+0.19}$ as found by the ATLAS experiment [50]. For more details about the theoretical conditions described here, we encourage the interested reader to consult Ref. [34].

\section{Mass spectra}

\section{Higgs-like ( $\mathcal{Z}_{2}$-even) scalars}

Expanding the scalar potential in terms of the VEVs and taking the second derivatives with respect to the scalar fields, one can obtain the mass terms and the mixing terms of the scalar fields. The SM Higgs is extracted from the mixing of three real scalars $h, \phi_{2}$, and $\delta_{3} .{ }^{1}$ The mixing matrix of these $\mathcal{Z}_{2}$-even neutral real scalars written on the basis of $\mathcal{S}=\left\{h, \phi_{2}, \delta_{3}\right\}^{\mathrm{T}}$ is given by

$$
\mathcal{M}_{0}^{2}=\left(\begin{array}{ccc}
2 \lambda_{H} v^{2} & \lambda_{H \Phi} v v_{\Phi} & \frac{v}{2}\left(M_{H \Delta}-2 \lambda_{H \Delta} v_{\Delta}\right) \\
\lambda_{H \Phi} v v_{\Phi} & 2 \lambda_{\Phi} v_{\Phi}^{2} & \frac{v_{\Phi}}{2}\left(M_{\Phi \Delta}-2 \lambda_{\Phi \Delta} v_{\Delta}\right) \\
\frac{v}{2}\left(M_{H \Delta}-2 \lambda_{H \Delta} v_{\Delta}\right) & \frac{v_{\Phi}}{2}\left(M_{\Phi \Delta}-2 \lambda_{\Phi \Delta} v_{\Delta}\right) & \frac{1}{4 v_{\Delta}}\left(8 \lambda_{\Delta} v_{\Delta}^{3}+M_{H \Delta} v^{2}+M_{\Phi \Delta} v_{\Phi}^{2}\right)
\end{array}\right) \text {. }
$$

The physical fields with definite mass can be obtained by doing the similarity transformation to this mixing matrix via orthogonal rotation matrix, $\mathcal{O}$, in such a way that

$$
\mathcal{O}^{\mathrm{T}} \cdot \mathcal{M}_{0}^{2} \cdot \mathcal{O}=\operatorname{Diag}\left(m_{h_{1}}^{2}, m_{h_{2}}^{2}, m_{h_{3}}^{2}\right),
$$

where the masses of the fields are arranged in ascending manner $m_{h_{1}} \leq m_{h_{2}} \leq m_{h_{3}}$. The interaction basis $\mathcal{S}$ and mass

\footnotetext{
${ }^{1}$ We follow the notations of [28] shifting the scalar fields as

$$
\begin{aligned}
H_{1} & =\left(\begin{array}{c}
G^{+} \\
\frac{v+h}{\sqrt{2}}+i G^{0}
\end{array}\right), \quad H_{2}=\left(\begin{array}{c}
H^{+} \\
H_{2}^{0}
\end{array}\right), \\
\Phi_{H} & =\left(\begin{array}{c}
G_{H}^{p} \\
\frac{v_{\Phi}+\phi_{2}}{\sqrt{2}}+i G_{H}^{0}
\end{array}\right), \quad \text { and } \quad \Delta_{H}=\left(\begin{array}{cc}
\frac{-v_{\Delta}+\delta_{3}}{2} & \frac{\Delta_{p}}{\sqrt{2}} \\
\frac{\Delta_{m}}{\sqrt{2}} & \frac{v_{\Delta}-\delta_{3}}{2}
\end{array}\right) .
\end{aligned}
$$
}

eigenstates are related through the $\mathcal{O}$ mixing matrix via $\mathcal{S}=\mathcal{O} \cdot\left\{h_{1}, h_{2}, h_{3}\right\}^{\mathrm{T}}$. In this setup, the $125 \mathrm{GeV}$ Higgs boson observed at the LHC is identified by the lightest mass eigenstate $h_{1}$.

Other $\mathcal{Z}_{2}$-even scalars are massless and would be Goldstone bosons $G^{ \pm, 0}$ and $G_{H}^{0}$ which do not mix with other scalar fields. However, they mix with the longitudinal components of the gauge fields and will be absorbed away.

\section{Dark ( $\mathcal{Z}_{2}$-odd) scalars}

The charged Higgs is sitting at the upper component of $\mathrm{H}_{2}$ which acquires mass from all three VEVs, but it does not mix with other fields. Since $\mathrm{H}_{2}$ couples to all three multiplets $H_{1}, \Phi_{H}$, and $\Delta_{H}$, after SSB it acquires tree mass terms one with each VEV given by 


$$
m_{H^{ \pm}}^{2}=M_{H \Delta} v_{\Delta}-\frac{1}{2} \lambda_{H}^{\prime} v^{2}+\frac{1}{2} \lambda_{H \Phi}^{\prime} v_{\Phi}^{2}
$$

The complex fields $G_{H}^{p, m}, H_{2}^{0(*)}$, and $\Delta_{p, m}{ }^{2}$ also acquire mass terms and mix. On the basis of $\mathcal{G}=\left\{G_{H}^{p}, H_{2}^{0 *}, \Delta_{p}\right\}^{T}$, the squared mass matrix is given by

$$
\mathcal{M}_{0}^{\prime 2}=\left(\begin{array}{ccc}
M_{\Phi \Delta} v_{\Delta}+\frac{1}{2} \lambda_{H \Phi}^{\prime} v^{2} & \frac{1}{2} \lambda_{H \Phi}^{\prime} v v_{\Phi} & -\frac{1}{2} M_{\Phi \Delta} v_{\Phi} \\
\frac{1}{2} \lambda_{H \Phi}^{\prime} v v_{\Phi} & M_{H \Delta} v_{\Delta}+\frac{1}{2} \lambda_{H \Phi}^{\prime} v_{\Phi}^{2} & \frac{1}{2} M_{H \Delta} v \\
-\frac{1}{2} M_{\Phi \Delta} v_{\Phi} & \frac{1}{2} M_{H \Delta} v & \frac{1}{4 v_{\Delta}}\left(M_{H \Delta} v^{2}+M_{\Phi \Delta} v_{\Phi}^{2}\right)
\end{array}\right)
$$

This matrix has zero determinant, which means that at least one of the mass eigenstates is massless. Despite complex fields, the mass matrix in Eq. (21) is real and symmetric; we can rotate this matrix into its diagonal form through a similarity transformation with the orthogonal matrix $\mathcal{O}^{D}$,

$$
\left(\mathcal{O}^{D}\right)^{\mathrm{T}} \cdot \mathcal{M}_{0}^{\prime 2} \cdot \mathcal{O}^{D}=\operatorname{Diag}\left(0, m_{D}^{2}, m_{\tilde{\Delta}}^{2}\right)
$$

The relation between interaction and mass states is given by $\mathcal{G}=\mathcal{O}^{D} \cdot\left\{\tilde{G}^{p}, D, \tilde{\Delta}\right\}^{\mathrm{T}}$. The first zero eigenvalue in Eq. (22) corresponds to $\tilde{G}^{p, m}$, the would-be Goldstone boson to be absorbed by $W^{\prime(p, m)}$, the complex gauge bosons of $S U(2)_{H}$. Here we assume the hierarchy $m_{D}^{2}<m_{\tilde{\Delta}}^{2}$. Note that we strictly avoid degenerate masses to simplify the analysis when $D$ is the dark matter candidate. However, from the mass expressions given below, one can see that very specific parameter choices are necessary to make the two massive states degenerate. The masses of the two physical massive eigenstates are given by

$$
M_{D, \tilde{\Delta}}^{2}=\frac{-B \mp \sqrt{B^{2}-4 A C}}{2 A},
$$

where

$$
\mathcal{M}_{1}^{2}=\left(\begin{array}{cccc}
\frac{g^{2} v^{2}}{4}+M_{Y}^{2} & -\frac{g^{\prime} g v^{2}}{4} & \frac{g^{\prime} g_{H} v^{2}}{4} & \frac{g^{\prime} g_{X} v^{2}}{2}+M_{X} M_{Y} \\
-\frac{g^{\prime} g v^{2}}{4} & \frac{g^{2} v^{2}}{4} & -\frac{g g_{H} v^{2}}{4} & -\frac{g g_{X} v^{2}}{2} \\
\frac{g^{\prime} g_{H} v^{2}}{4} & -\frac{g g_{H} v^{2}}{4} & \frac{g_{H}^{2}\left(v^{2}+v_{\Phi}^{2}\right)}{4} & \frac{g_{H} g_{X}\left(v^{2}-v_{\Phi}^{2}\right)}{2} \\
\frac{g^{\prime} g_{X} v^{2}}{2}+M_{X} M_{Y} & -\frac{g g_{X} v^{2}}{2} & \frac{g_{H} g_{X}\left(v^{2}-v_{\Phi}^{2}\right)}{2} & g_{X}^{2}\left(v^{2}+v_{\Phi}^{2}\right)+M_{X}^{2}
\end{array}\right),
$$

$$
\begin{aligned}
A= & 8 v_{\Delta}, \\
B= & -2\left[M_{H \Delta}\left(v^{2}+4 v_{\Delta}^{2}\right)+M_{\Phi \Delta}\left(4 v_{\Delta}^{2}+v_{\Phi}^{2}\right)\right. \\
& \left.+2 \lambda_{H \Phi}^{\prime} v_{\Delta}\left(v^{2}+v_{\Phi}^{2}\right)\right], \\
C= & \left(v^{2}+v_{\Phi}^{2}+4 v_{\Delta}^{2}\right)\left[M_{H \Delta}\left(\lambda_{H \Phi}^{\prime} v^{2}+2 M_{\Phi \Delta} v_{\Delta}\right)\right. \\
& \left.+\lambda_{H \Phi}^{\prime} M_{\Phi \Delta} v_{\Phi}^{2}\right] .
\end{aligned}
$$

The lightest state between $H^{ \pm}$and $D$, if lighter than every other $\mathcal{Z}_{2}$-odd state, has the possibility to become the DM candidate. However, an electrically charged DM candidate such as $H^{ \pm}$is undesirable. For this reason, we will concentrate on parameter space where $m_{H^{ \pm}}>m_{D}$.

\section{Gauge bosons}

After SSB, the gauge bosons that acquire mass terms are the $B, X$ and all the components of $W$ and $W^{\prime}$. The charged $W^{ \pm}$gauge bosons remains completely SM-like with its mass given by $M_{W}=g v / 2$. The $W^{\prime p}=\left(W^{\prime m}\right)^{*}$ does not mix with the SM $W^{ \pm}$and acquires a mass given by

$$
m_{W^{\prime}(p, m)}^{2}=\frac{1}{4} g_{H}^{2}\left(v^{2}+v_{\Phi}^{2}+4 v_{\Delta}^{2}\right) .
$$

The remaining gauge bosons, $B, W^{3}, W^{\prime 3}$, and $X$ have mixing terms. We can write their mass terms as a $4 \times 4$ matrix using the basis $\mathcal{V}^{\prime}=\left\{B, W^{3}, W^{\prime 3}, X\right\}^{\mathrm{T}}$,

where $M_{X}$ and $M_{Y}$ are the two Stueckelberg mass parameters [51-59] introduced for $U(1)_{X}$ and $U(1)_{Y}$, respectively. This mass matrix has zero determinant, meaning that there is at least one massless state that can be identified with

\footnotetext{
${ }^{2}$ See previous footnote for the definitions of these complex scalars.
} 
the photon. The remaining three states are massive in general. One of them, the $Z$, is related to the SM gauge boson $Z^{\mathrm{SM}}$, and the other two are the extra gauge bosons $Z^{\prime}$ and $Z^{\prime \prime}$. As in the neutral scalars case, we can diagonalize this mass matrix by an orthogonal rotation matrix $\mathcal{O}_{4 \times 4}^{G}$ such that $\mathcal{V}^{\prime}=\mathcal{O}_{4 \times 4}^{G} \cdot\left\{A, Z, Z^{\prime}, Z^{\prime \prime}\right\}^{\mathrm{T}}$. We will also use $Z_{i}$ with $i=1,2,3$ for $Z, Z^{\prime}, Z^{\prime \prime}$, respectively, in the following. As noted in Ref. [35], one can justify the parameter choice $M_{Y}=0$ by considering the electric charges of the fermions. Otherwise, the neutrinos would not be neutral and all the SM electric charges would receive a correction that grows with $M_{Y}$. Therefore, hereafter we will consider $M_{Y}=0{ }^{3}$
This choice makes possible to rotate the first and second rows and columns of Eq. (26) (with $M_{Y}$ set to 0) using the Weinberg angle in a SM-like manner. Applying the $4 \times 4$ rotation

$$
\mathcal{O}^{W}=\left(\begin{array}{cccc}
c_{W} & -s_{W} & 0 & 0 \\
s_{W} & c_{W} & 0 & 0 \\
0 & 0 & 1 & 0 \\
0 & 0 & 0 & 1
\end{array}\right)
$$

as $\left(\mathcal{O}^{W}\right)^{\mathrm{T}} \cdot \mathcal{M}_{1}^{2}\left(M_{Y}=0\right) \cdot \mathcal{O}^{W}$, we find the mass matrix

$$
\mathcal{M}_{Z}^{2}=\left(\begin{array}{cccc}
0 & 0 & 0 & 0 \\
0 & M_{Z^{\mathrm{SM}}}^{2} & -\frac{g_{H} v}{2} M_{Z^{\mathrm{SM}}} & -g_{X} v M_{Z^{\mathrm{SM}}} \\
0 & -\frac{g_{H} v}{2} M_{Z^{\mathrm{SM}}} & \frac{g_{H}^{2}\left(v^{2}+v_{\Phi}^{2}\right)}{4} & \frac{g_{X} g_{H}\left(v^{2}-v_{\Phi}^{2}\right)}{2} \\
0 & -g_{X} v M_{Z^{\mathrm{SM}}} & \frac{g_{X} g_{H}\left(v^{2}-v_{\Phi}^{2}\right)}{2} & g_{X}^{2}\left(v^{2}+v_{\Phi}^{2}\right)+M_{X}^{2}
\end{array}\right),
$$

where $M_{Z^{\mathrm{SM}}}=\sqrt{g^{2}+g^{\prime 2}} v / 2$ is the SM gauge boson $Z^{\mathrm{SM}}$ mass. Given the form of the rotation matrix $\mathcal{O}^{W}$, we can identify the first component of the basis of this matrix with the photon, which is immediately massless, and the second with the $Z^{\mathrm{SM}}$. The new intermediate basis in this case is $\mathcal{V}_{Z}^{\prime}=\left\{A, Z^{\mathrm{SM}}, W^{\prime 3}, X\right\}^{\mathrm{T}}$. We can rewrite the original rotation matrix as the product of two matrices $\mathcal{O}_{4 \times 4}^{G}\left(M_{Y}=0\right)=\mathcal{O}^{W} \cdot \mathcal{O}^{Z}$, where the matrix $\mathcal{O}^{Z}$ diagonalizes $\mathcal{M}_{Z}^{2}$ in Eq. (28). In that case, we can relate the mass eigenstates with the intermediate states as $\mathcal{V}_{Z}^{\prime}=\mathcal{O}^{Z}$. $\left\{A, Z, Z^{\prime}, Z^{\prime \prime}\right\}^{\mathrm{T}}$. Hereafter, we will call $\mathcal{O}^{G}$ to the nondiagonal $3 \times 3$ part of $\mathcal{O}^{Z}$, such that $\mathcal{O}_{j+1, k+1}^{Z}=\mathcal{O}_{j, k}^{G}$ with $j$ and $k=1,2,3$, as explicitly shown in Eq. (6) of Ref. [35]. Note that the photon $A$ remains the same between the intermediate states $\mathcal{V}_{Z}^{\prime}$ and the mass eigenstates. This necessarily means that the only nonzero element in the first column and row of $\mathcal{O}^{Z}$ is $\mathcal{O}_{1,1}^{Z}=1$.

Interestingly, the only gauge boson that acquires mass contributions from the three nonzero VEVs is the $W^{\prime(p, m)}$ with its mass given in Eq. (25).

\section{ACCIDENTAL DISCRETE SYMMETRY ( $h$ PARITY) AND DARK MATTER CANDIDATE}

As mentioned in the previous section, the stability of the scalar dark matter candidate in this model is protected by the accidental discrete $\mathcal{Z}_{2}$ symmetry in the scalar potential which is automatically implied by the $S U(2)_{L} \times U(1)_{Y} \times$ $S U(2)_{H} \times U(1)_{X}$ gauge symmetry. Due to its special vacuum alignment where the $\mathrm{H}_{2}$ field does not acquire a VEV, the accidental $\mathcal{Z}_{2}$ symmetry remains intact after SSB. It was argued in [28] that there is no gauge invariant higher dimensional operator that one can write down which can lead to the decay of DM candidate in G2HDM. The presence of the accidental discrete $\mathcal{Z}_{2}$ symmetry after SSB reinforces such argument.

This discrete $\mathcal{Z}_{2}$ symmetry in G2HDM that we observe here is kind of peculiar in the sense that different components of the $S U(2)_{H}$ doublets $H$ and $\Phi_{H}$, and triplet $\Delta_{H}$ have opposite parity. Thus, for dark matter physics, it is mandatory to give VEVs to those scalars with even parity. Otherwise, the $\mathcal{Z}_{2}$ symmetry will be broken spontaneously which will lead to no stable DM as well as the domain wall problem in the early Universe. Another peculiar feature of this $\mathcal{Z}_{2}$ symmetry is that it acts on the complex fields. We will refer this accidental discrete $\mathcal{Z}_{2}$ symmetry as the hidden parity ( $h$ parity) in G2HDM in what follows.

This $h$ parity can actually be extended to the whole renormalizable Lagrangian of G2HDM, including the gauge, scalar, and Yukawa interactions. For example, while the SM $W^{ \pm}$and all the neutral gauge bosons $\gamma, Z_{i}$ are always coupled to a pair of SM fermions $\bar{f} f^{(\prime)}$ or a pair of new heavy fermions $\bar{f}^{H} f^{(\prime) H}$, the $W^{\prime(p, m)}$ always couples to one SM fermion and one new heavy fermion $\bar{f}^{H} f^{\prime}$ or $\bar{f} f^{\prime H}$. Similar features can be observed in the gauge-Higgs sector and the Yukawa couplings in G2HDM. For instance, while $\gamma, Z_{i}$, and $W^{ \pm}$are always coupled to a pair of $\mathcal{Z}_{2}$-even scalars or a pair of $\mathcal{Z}_{2}$-odd scalars, the $W^{\prime(p, m)}$ always couples to one $\mathcal{Z}_{2}$-even and one $\mathcal{Z}_{2}$-odd scalars. Also, $h_{i}$

\footnotetext{
${ }^{3}$ Note that with both vanishing $M_{X}$ and $M_{Y}$, the mass matrix in Eq. (26) will have two zero eigenvalues and one would enjoy to have two unbroken $U(1)$ s. One of them is the familiar photon, while the other can be identified as the dark photon. With just one nonzero $M_{X}$, the remaining local symmetry is the electromagnetism $U(1)_{\mathrm{EM}}$.
} 
TABLE II. Classification of all the fields in G2HDM under $h$ parity.

\begin{tabular}{lr}
\hline \hline Fields & $h$ parity \\
\hline$h, G^{ \pm, 0}, \phi_{2}, G_{H}^{0}, \delta_{3}, f, W_{1,2,3}^{\mu}, B_{\mu}, X^{\mu}, W_{3}^{\mu \prime}, G^{\mu a}$ & 1 \\
$G_{H}^{p, m}, H_{2}^{0}, H_{2}^{0 *}, H^{ \pm}, \Delta_{p, m}, f^{H}, W_{1,2}^{\mu^{\prime}}$ & -1 \\
\hline \hline
\end{tabular}

always couples to either $\bar{f} f$ or $\bar{f}^{H} f^{H}$, while for the new Yukawa couplings, the dark matter $D\left(D^{*}\right)$ always couples with $\bar{u} u^{H}$ and $\bar{d}^{H} d\left(\bar{u}^{H} u\right.$ and $\left.\bar{d} d^{H}\right)$ and the charged Higgs $H^{+}\left(H^{-}\right)$always couples with $\bar{u} d^{H}$ and $\bar{u}^{H} d\left(\bar{d}^{H} u\right.$ and $\left.\bar{d} u^{H}\right)$, etc. Therefore, besides the $\mathcal{Z}_{2}$-even/-odd scalars discussed in the previous section, one is naturally led to assign $W^{\prime(p, m)}$ and all new heavy fermions $f^{H}$ to have odd $h$ parity, and all SM gauge particles including the additional neutral gauge bosons to have even $h$ parity. A summary of the $h$ parity for all the fields in G2HDM is collected in Table II.

Thus, besides the two well-known accidental global symmetries of baryon number and lepton number inherited from the SM, there is also an accidental discrete $\mathcal{Z}_{2}$ symmetry in G2HDM. Other than protecting the stability of the lightest electrically neutral $\mathcal{Z}_{2}$-odd particle to give rise to a DM candidate, this accidental $\mathcal{Z}_{2}$ symmetry also provides natural flavor conservation laws for neutral currents $[60,61]$ at the tree level for the SM sector in G2HDM [28], as described in the previous paragraph. While it is important to unravel if the $h$ parity in G2HDM has a deeper origin from a larger theoretical structure, for example, like grand unification or supersymmetry or brane world, we will not pursue further here.

In principle, any electrically neutral $\mathcal{Z}_{2}$-odd neutral particle can be a DM candidate (e.g., the heavy neutrinos $\nu^{H}$, the complex scalar mass eigenstate $D$, and the gauge boson $\left.W^{\prime(p, m)}\right)$. In this work, we focus on the lightest $\mathcal{Z}_{2^{-}}$ odd complex scalar field $D$. From Eq. (21), we know that $D$ is a linear combination of the interaction states $G_{H}^{p}, H_{2}^{0 *}$, and $\Delta_{p}$. Using $\mathcal{G}=\mathcal{O}^{D} \cdot\left\{\tilde{G}^{p}, D, \tilde{\Delta}\right\}^{\mathrm{T}}$, we can write this linear combination as

$$
D=\mathcal{O}_{12}^{D} G_{H}^{p}+\mathcal{O}_{22}^{D} H_{2}^{0 *}+\mathcal{O}_{32}^{D} \Delta_{p},
$$

where $\mathcal{O}_{i j}^{D}$ represents the $(i, j)$ element of the orthogonal matrix $\mathcal{O}^{D}$. The actual values of the elements of this matrix depend on the actual numerical values of the parameters in Eq. (21).

Since a particular dominant component cannot be inferred from Eq. (21) together with the constraints presented in Sec. II B, we take the approach of considering three different main compositions. Using the rotation matrix elements, we can define the $G_{H}^{p}, H_{2}^{0 *}$, and $\Delta_{p}$ compositions as $f_{G^{p}}=\left(\mathcal{O}_{12}^{D}\right)^{2}, f_{H_{2}}=\left(\mathcal{O}_{22}^{D}\right)^{2}$, and $f_{\Delta_{p}}=\left(\mathcal{O}_{32}^{D}\right)^{2}$, respectively, satisfying $f_{G^{p}}+f_{H_{2}}+f_{\Delta_{p}}=1$. Our results will be classified in three different cases as follows:

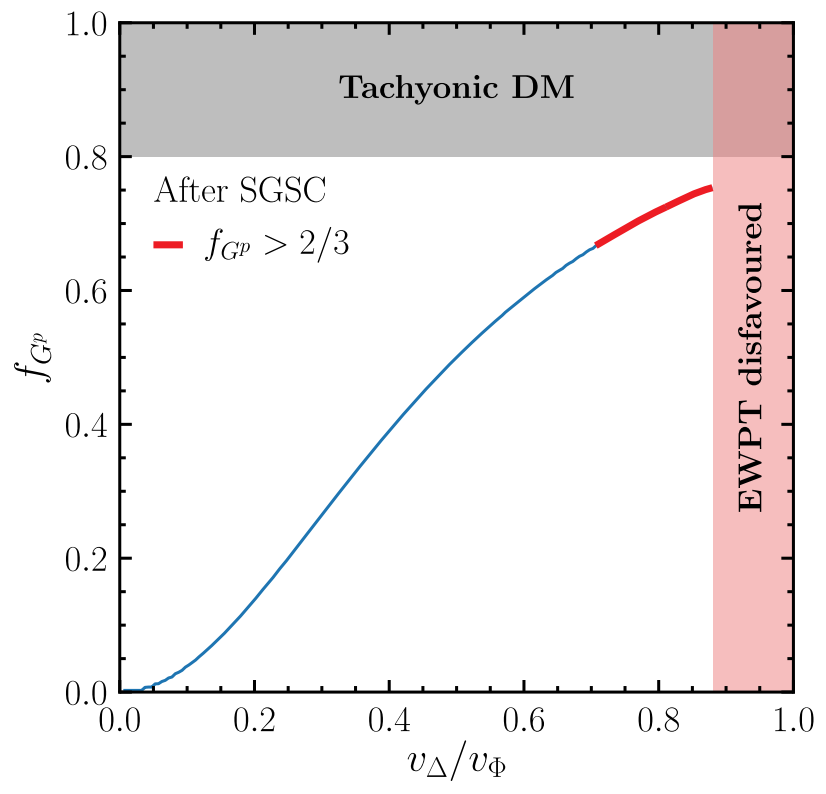

FIG. 1. Correlation between the ratio $v_{\Delta} / v_{\Phi}$ and the composition mixing parameter $f_{G^{p}}$ for all the DM types after applying constraints from the scalar and gauge sectors.

(1) Inert doubletlike DM for $f_{H_{2}}>2 / 3$.

(2) $S U(2)_{H}$ tripletlike DM for $f_{\Delta_{p}}>2 / 3$.

(3) $S U(2)_{H}$ Goldstone bosonlike DM for $f_{G^{p}}>2 / 3$.

To avoid cluttering in the following, we will use the more concise terms doubletlike, tripletlike, and Goldstone-like DM to refer to the above cases of 1,2, and 3, respectively.

In order to realize any one of the three cases of the DM discussed above, one needs to have its diagonal element in the mass matrix given by Eq. (21) to be the lightest, while its mixings with the other two off-diagonal elements are small. However, the mixing among the other two can be arbitrary. Take the Goldstone-like DM as an example. It is easy to note that the $(1,1)$ and $(3,3)$ elements of the mass matrix in Eq. (21) have a see-saw behavior controlled by the value of $v_{\Delta}$. The $(2,2)$ element remains almost unaffected thanks to the term proportional to large $v_{\Phi}^{2}$. Goldstone-like DM is characterized by a large value in the $(1,1)$ element of Eq. $(21)$ when compared to the $(1,2)$ and $(1,3)$ elements, given by $\lambda_{H \Phi}^{\prime} v v_{\Phi} / 2$ and $-M_{\Phi \Delta} v_{\Phi} / 2$, respectively, so as to suppress the mixing effects. The size of the $(1,2)$ element is not relevant since it is proportional to the smaller term $v v_{\Phi}$ as compared with both the $(1,1)$ and $(2,2)$ elements which are always much larger. The difference in size between the $(1,1)$ and $(1,3)$ elements is best measured by taking the ratio between them, which is roughly about $2 v_{\Delta} / v_{\Phi}$. In other words, the $v_{\Delta} / v_{\Phi}$ ratio controls the Goldstone boson composition of the DM mass eigenstate. This is illustrated in Fig. 1, where the correlation between the ratio $v_{\Delta} / v_{\Phi}$ and the composition mixing parameter $f_{G^{p}}$ is shown for all DM types. The small arc in the correlation curve with $f_{G^{p}}>2 / 3$ is highlighted by red color, indicating only a small parameter space is 
allowed for Goldstone-like DM. Note that when the ratio $v_{\Delta} / v_{\Phi}$ grows close to 1, EWPT disfavors the presence of relatively light $Z^{\prime}$ state with larger mixing with the SM $Z$. Values of $f_{G^{p}}$ larger than $\sim 0.8$ are accessible only through negative $M_{\Phi \Delta}$, resulting in tachyonic DM mass. Therefore, to realize a Goldstone-like DM with $f_{G^{p}}>2 / 3$ in the following numerical analysis, one needs to do some finetunings in the parameter space. For the inert doubletlike and tripletlike DM, no fine-tunings are required.

\section{DARK MATTER EXPERIMENTAL CONSTRAINTS}

To determine WIMP DM properties, one can measure the DM-SM interactions via several approaches. Conventionally, DM direct detection, DM indirect detection, and collider search are used for the hunting for DM. Thus far, null signals were reported from all these experimental efforts and only allowed regions have been shown in the DM model parameter space. On the other hand, the DM relic density measurement can indeed give a signal region which can constrain the parameter space of DM model in a significant way. Hence, our strategy is to determine the parameter space in G2HDM allowed by the current relic density measurement, and the limits deduced from DM direct detection, DM indirect detection, and collider search (at the LHC). In this section, we briefly describe each of these experimental constraints used in this analysis.

\section{A. Relic density}

It is fascinating to wonder about the thermal history of DM based on all our current knowledge of physics. The simplest scenario is that a WIMP maintains its thermal equilibrium with the SM sector before freeze-out and the DM number density can be described by a Boltzmann distribution. Therefore, the DM mass determines its abundance before freeze-out. As in most WIMP theories, owing to the small DM-SM couplings, the relic density comes out too large and the correct abundance can be only achieved by some specific mechanisms. The mechanisms to reduce the thermal DM relic density in the G2HDM can be both from DM annihilation and also from coannihilation with heavier $\mathcal{Z}_{2}$-odd particles. Coannihilation only happens if the next lightest $\mathcal{Z}_{2}$-odd particles are slightly heavier than DM (usually $\lesssim 10 \%$ ) so that its number density at the temperature higher than freeze-out does not suffer a large Boltzmann suppression. In our setup, the heavier $\mathcal{Z}_{2}$-odd scalar $\tilde{\Delta}$, the charged Higgs, new heavy fermions, or gauge boson $W^{\prime(p, m)}$ can coannihilate with the DM candidate $D$. Additionally, the SM Higgs and $Z$ resonance can play an important role for the doubletlike DM while there is no $Z$ resonance in the tripletlike and Goldstone-like DM cases because both $\Delta_{H}$ and $\Phi_{H}$ are SM singlets. As we will see later, the couplings between DM and some of the mediators in G2HDM could be suppressed by mixings or cancellations.
The scalar $\tilde{\Delta}$ and the DM candidate $D$ come from the same mass matrix. The splitting between their masses is mostly controlled by the second term in the numerator of Eq. (23). Hence, coannihilation between DM and $\tilde{\Delta}$ can only happen if $M_{\Phi \Delta} \gtrsim \mathcal{O}(10 \mathrm{GeV})$ and $v_{\Phi} \gtrsim 70 \mathrm{TeV}$. However, this condition also makes DM masses at around $\mathcal{O}(1 \mathrm{TeV})$ or larger.

For the doubletlike DM case, the mass of the DM candidate is close to the mass of the charged Higgs with the splitting approximately given by

$$
m_{H^{ \pm}}^{2}-m_{D}^{2} \approx-\frac{1}{2} \lambda_{H}^{\prime} v^{2}
$$

in the approximation where DM mass is dominated by the $(2,2)$ element of the matrix in Eq. (21). For tripletlike DM, the mass differences between $D$ and the other heavy $\mathcal{Z}_{2^{-}}$ odd scalars are usually large enough so that coannihilation can be avoided. Coannihilation between $D$ and $W^{\prime}$ occurs for DM mass closing to $W^{\prime}$ mass which is heavy due to large $v_{\Phi}$. As for the resonance, only $h_{1}$ and $h_{2}$ resonances are present. Due to $\Delta_{H}$ being an SM singlet, any $D$ annihilation through $Z$-boson like mediator is suppressed by mixings.

To compare against experimental data, we will consider the latest result from the PLANCK Collaboration [62] for the relic density, $\Omega h^{2}=0.120 \pm 0.001$. In particular, we will require the parameter space of G2HDM to reproduce this well-measured value with a $2 \sigma$ significance.

\section{B. Direct detection}

The most recent constraint for DM direct search is given by the XENON1T Collaboration [63]. The null signal result from this search puts the most stringent limit on DMnucleon cross section so far, especially for the DM mass that lies between 10 and $100 \mathrm{GeV}$. The XENON1T Collaboration excluded DM-nucleon elastic cross sections above $10^{-46} \mathrm{~cm}^{2}$ for a DM particle with mass around $25 \mathrm{GeV}$.

In models with isospin violation (ISV), DM interactions with proton and neutron can be different and the ratio between the DM-neutron and DM-proton effective couplings, $f_{n} / f_{p}$, can have values that differ from 1 significantly depending on the model parameters. In particular, for a target made of xenon, the ratio $f_{n} / f_{p} \approx-0.7$ corresponds to maximal cancellation between proton and neutron contributions [64].

For instance, if DM interacts with nucleons mediated by the $Z$ boson, the strength is characterized by the electric charge and the third generator $T_{3}$ of $S U(2)_{L}$ group. The vectorial coupling of quark $q$ ( $u$ or $d$ type) to the SM $Z$ boson in G2HDM is

$g_{\bar{q} q Z}^{V}=\frac{i}{2}\left[\frac{g}{c_{W}}\left(T_{3}-2 Q_{q} s_{W}^{2}\right) \mathcal{O}_{11}^{G}+g_{H} T_{3}^{\prime} \mathcal{O}_{21}^{G}+g_{X} X \mathcal{O}_{31}^{G}\right]$. 
Due to different $Q_{q}, T_{3}, T_{3}^{\prime}$, and $X$ charges, this coupling is expected to vary depending on the quark $q$ being $u$ or $d$ type. Hence, the $Z$ boson interacts with proton and neutron differently and the $f_{n} / f_{p}$ can be different from 1 . For the case of DM with a non-negligible doublet composition, we found that DM can couple to proton or neutron differently via $Z_{i}$ boson exchange and it leads to ISV.

Generally speaking, exact cancellation between neutrons and protons is expected to be in a tiny region of parameter space. Nevertheless, the G2HDM doubletlike DM can have a much wider distribution of $f_{n} / f_{p}$. Furthermore, a more subtle interference between the contributions from neutral gauge and Higgs boson exchange can result in two different scattering cross sections for $\mathrm{DM}(D)$ and anti-DM $\left(D^{*}\right)$ with neutrons. Such a difference may compensate for any cancellation caused by ISV. While one can expect a negligible cancellation from ISV effects in most of the G2HDM parameter space, we consider these effects in all the DM scenarios. To include ISV effects, one has to compute the DM-nucleus elastic scattering cross section $\sigma_{\mathcal{D N}}$,

$$
\sigma_{D \mathcal{N}}=\frac{4 \mu_{\mathcal{A}}^{2}}{\pi}\left[f_{p} \mathcal{Z}+f_{n}(\mathcal{A}-\mathcal{Z})\right]^{2},
$$

where $\mathcal{N}$ stands for a nucleus with mass number $\mathcal{A}$ and proton number $\mathcal{Z}$. For definiteness, we will ignore all the isotopes of xenon and fix $\mathcal{A}$ and $\mathcal{Z}$ to 131 and 54, respectively, in this work. We obtain the effective couplings $f_{p}$ and $f_{n}$ by using micromEGAs [65]. The DM-nucleon reduced mass is denoted as $\mu_{\mathcal{A}}=m_{D} m_{\mathcal{A}} /\left(m_{D}+m_{\mathcal{A}}\right)$. On the other hand, the limit published by XENON1T is for the nucleon with isospin conserving assumption $f_{n}=f_{p}$. To reconstruct the XENON1T results at the nucleus level for general value of the ratio $f_{n} / f_{p}$, we use the following expression:

$$
\sigma_{D \mathcal{N}}^{\mathrm{X} 1 \mathrm{~T}}=\sigma_{p}^{\mathrm{SI}}(\mathrm{X} 1 \mathrm{~T}) \times \frac{\mu_{\mathcal{A}}^{2}}{\mu_{p}^{2}} \times\left[\mathcal{Z}+\frac{f_{n}}{f_{p}}(\mathcal{A}-\mathcal{Z})\right]^{2},
$$

where $\mu_{p}^{2}$ is the DM-proton reduced mass. In this work, we use Eq. (33) to constrain our direct detection prediction.

Since we are dealing with complex scalar DM, we need to consider the anti-DM interaction with the nucleon. The DM-nucleon interaction and anti-DM-nucleon interaction in general can be quite different. When the mediators are heavy enough, one can integrate them out to obtain effective interactions for the DM and nucleon. The spin independent interaction for complex scalar DM can be written in terms of effective operator as [66]

$$
\mathcal{L}_{D}=2 \lambda_{N, e} M_{D} D D^{*} \bar{\psi}_{N} \psi_{N}+i \lambda_{N, o}\left(D^{*}{\stackrel{\leftrightarrow}{\partial_{\mu}}} D\right) \bar{\psi}_{N} \gamma^{\mu} \psi_{N},
$$

where the $\psi_{N}, \lambda_{N, e}$, and $\lambda_{N, o}$ denote the nucleon field operator, the coupling of even operator, and the coupling of

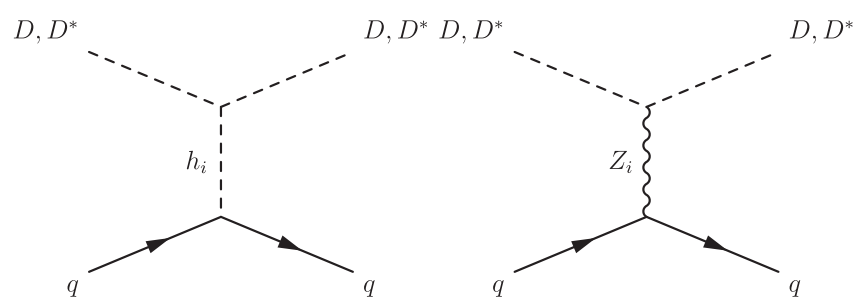

FIG. 2. The dominant Feynman diagrams with the $\mathcal{Z}_{2}$-even Higgs bosons (left) and neutral gauge bosons (right) exchange for direct detection of DM.

odd operator, respectively. The effective coupling of DM (anti-DM) with the nucleon is given by

$$
\lambda_{N}=\frac{\lambda_{N, e} \pm \lambda_{N, o}}{2},
$$

where the plus (minus) sign stands for DM-nucleon (antiDM-nucleon) interaction. The first term in the right-hand side of Eq. (34) represents the even operator interaction between DM and the nucleon. It is called even operator because when one exchanges $D$ with $D^{*}$, the interaction stays the same. On the other hand, under a similar exchange between $D$ and $D^{*}$, the second term flips sign. Thus, it is called odd operator. As a result, the interaction strength between DM-nucleon and anti-DM-nucleon will not be the same and it is given by Eq. (35). Hence, the numerical value of $\sigma_{D^{*} \mathcal{N}}$ is in general not equal to $\sigma_{D \mathcal{N}}$ given by Eq. (32) because the effective couplings $f_{p}$ and $f_{n}$ for $D$ are not the same as those for $D^{*}$.

The Feynman diagrams of the dominant contribution to describe DM-quark interactions in the G2HDM are shown in Fig. 2. The left panel is the $t$ channel with three Higgs bosons exchange, while the right panel is the $t$ channel with three neutral gauge bosons exchange. Thus, G2HDM captures the features from both the Higgs-portal and vector-portal DM models in the literature.

Note that the doubletlike DM in this model has a large scattering cross section because the vertex $D D^{*} Z$ is governed by the SM coupling as shown in Eq. (A2) and hence not suppressed by any mixing angle. Due to the additional contributions from other heavy gauge bosons $\left(Z^{\prime}\right.$ and $Z^{\prime \prime}$ ), the DM-neutron cross section is 3 orders of magnitude larger than the DM-proton cross section. This ISV effect is also observed in tripletlike $\operatorname{DM}\left(\Delta_{p}\right)$, but the ISV occurs mildly due to the mixing suppression between the DM $\left(\Delta_{p}\right)$ and $Z$ coupling. This suppression makes the $Z$ exchange contribution comparable with $Z^{\prime}$ and $Z^{\prime \prime}$, as well as the contribution from the SM Higgs $h_{1}$ exchange.

\section{Indirect detection: gamma ray from dSphs}

Excluding the early Universe, DM at the present may also annihilate to SM particles significantly at the halo center where DM density is dense enough to produce 
cosmic rays or photons which can be distinguished from those standard astrophysical background. Such a measurement is known as DM indirect detection. As long as indirect detection constraints are concerned, the continuum gammaray observations from dwarf spheroidal galaxies (dSphs) can usually place a robust and severe limit on the DM annihilation cross section for DM masses larger than $10 \mathrm{GeV}$ [67]. This is owing to two advantages of searching DM at the dSphs. First, the dSphs provides an almost background-free system because they are faint but widely believed to be DM dominated systems. Second, their kinematics can be precisely measured; hence, the systematical uncertainties from DM halo can be controlled. Therefore, in this work, we will only use the dSphs constraints implemented in LikeDM [68] to evaluate the $\chi^{2}$ statistics of our model based on Fermi Pass 8 data (photon counts), recorded from August 4, 2008 to August 4,2015 . The two-dimensional $2 \sigma$ criteria is taken to be $\Delta \chi^{2}=5.99$ in our study.

The standard gamma-ray fluxes produced from DM annihilation at the dSphs halo is given by

$$
\frac{d \Phi_{\gamma}}{d E_{\gamma}}=\frac{\langle\sigma v\rangle}{8 \pi m_{D}^{2}} \times J \times \sum_{\mathrm{ch}} \mathrm{BR}(\mathrm{ch}) \times \frac{d N_{\gamma}^{\mathrm{ch}}}{d E_{\gamma}},
$$

where $J=\int d l d \Omega \rho(l)^{2}$ is the so-called $J$ factor, which integrates along the line-of-sight $l$ with the telescope opening angle given by $\Omega$. The DM density distribution is denoted as $\rho(l)$. Here, we take $15 \mathrm{dSphs}$ and their $J$ factors as the default implementation in LikeDM. The index ch runs over all the DM annihilation channels. The annihilation branching ratio $\mathrm{BR}(\mathrm{ch})$ and energy spectra $d N_{\gamma}^{\mathrm{ch}} / d E_{\gamma}$ are computed by using microMEGAs and PPPC4 [69], respectively.

Similar to Higgs portal models, an inert Higgs DM in our setup can only annihilate to the SM fermions via Higgs portal or $Z$ boson. On the other hand, if the DM is heavier than $m_{W}$, then the four points interaction $D D^{*} W^{+} W^{-}$can have a higher photon flux to be tested.

\section{Collider search}

\section{Monojet search}

DM particles could be produced copiously at colliders. Unfortunately, DM cannot be detected on its own since it would pass through detectors without leaving any trace. Therefore, one should look for the DM production associated with visible SM particles. At the LHC, the signal of an energetic jet from initial state radiation that balances the momentum of undetected DM, usually referred to monojet signal, is one of the sensitive channels to the search for DM. As shown in Fig. 3, the DM pairs are mainly produced in the Feynman diagrams with the exchanges of $\mathcal{Z}_{2}$-even Higgs bosons and neutral gauge bosons in the G2HDM. For numerical study, we take the parameters allowed by EWPT [35] and the XENON1T constraints (to be discussed

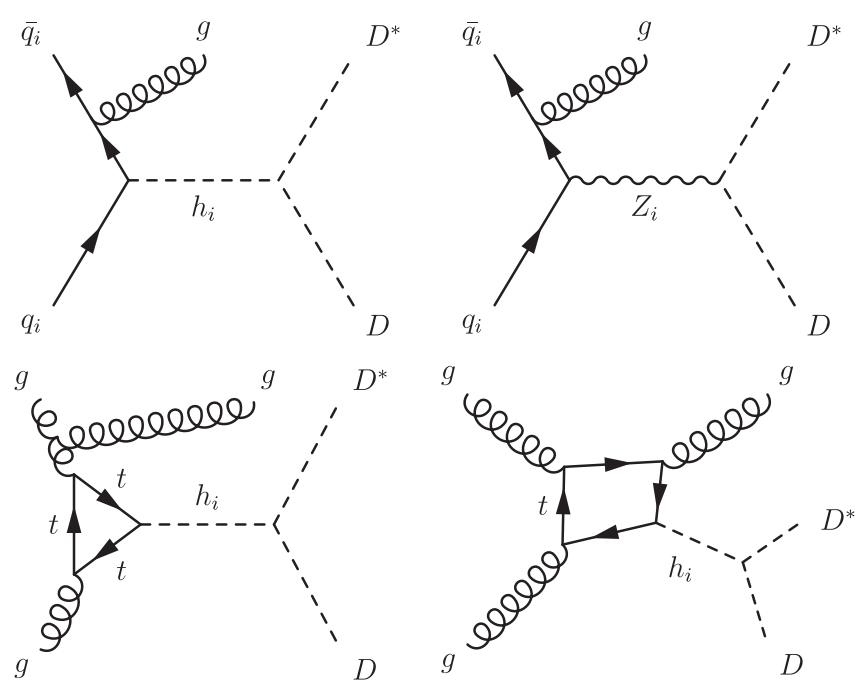

FIG. 3. The representative Feynman diagrams of leading contributions for monojet at the LHC in the G2HDM, where $h_{i}=h_{1}, h_{2}, h_{3}$ and $Z_{i}=Z, Z^{\prime}, Z^{\prime \prime}$.

in Sec. V) and find out that the cross sections are far below the current limits set by ATLAS [70] and CMS [71] Collaborations at the LHC. Therefore, the monojet search would not play any significant role in determining the viable parameter space for DM in G2HDM.

\section{Invisible Higgs decay}

The Higgs boson will decay into a pair of DMs when the DM is lighter than half its mass. This decay channel is known as the invisible decay of the Higgs boson. At tree level in G2HDM, the partial decay width of the Higgs boson to pair of dark matter, $h_{1} \rightarrow D D^{*}$, is given by

$$
\Gamma\left(h_{1} \rightarrow D D^{*}\right)=\frac{\left(\mathcal{O}_{32}^{D}\right)^{4}}{16 \pi m_{h_{1}}} \lambda_{D D^{*} h_{1}}^{2} \sqrt{1-\frac{4 m_{D}^{2}}{m_{h_{1}}^{2}}},
$$

where the $\lambda_{D D^{*} h_{1}}$ coupling depends on the composition of the $h_{1}$. For example, for the tripletlike DM case, it can be deduced from Eq. (A6), viz.,

$$
\lambda_{D D^{*} h_{1}} \approx \mathcal{O}_{11} \lambda_{H \Delta} v+\mathcal{O}_{21} \lambda_{\Phi \Delta} v_{\Phi}-2 \mathcal{O}_{31} \lambda_{\Delta} v_{\Delta}
$$

Currently, the upper limit on the Higgs invisible decay branching ratio is rather loose, about $24 \%$ at $95 \%$ C.L. [72] at the LHC. Taking $m_{D} \ll m_{h_{1}}$ together with SM Higgs total decay width of $13 \mathrm{MeV}$ [72], the LHC limit implies an upper bound,

$$
\left(\mathcal{O}_{32}^{D}\right)^{2} \lambda_{D D^{*} h_{1}}<5.099 \mathrm{GeV}
$$

However, we found this limit is not as stringent as DM direct detection unless $m_{D} \lesssim 10 \mathrm{GeV}$ where $\mathrm{DM}$ recoil energy is below the XENON1T threshold. 


\section{NUMERICAL ANALYSIS AND RESULTS}

\section{A. Methodology}

In order to keep consistency with previous G2HDM studies, in particular the scalar sector constrains presented in [34], we will perform random scans to generate a sample of points consistent with all the conditions mentioned there. In our case, we will not keep $v_{\Phi}$ fixed. Due to $Z^{\prime}$ search constraints [35], we start our scan range at $v_{\Phi}=20 \mathrm{TeV}$. Considering the energy scale for future colliders, we scan $v_{\Phi}$ up to $100 \mathrm{TeV}^{4}$

We will complete the scan with the free parameters of the gauge sector $g_{H}$ and $g_{X}$, while fixing the Stueckelberg mass parameter $M_{X}=2 \mathrm{TeV}$ corresponds to the heavy $M_{X}$ scenario discussed in [35]. We will keep the $g_{H}$ coupling below 0.1 to avoid the Drell-Yan constraints. The lower bound of $g_{H}$ will be decided point by point such that the $W^{\prime}$ boson is heavier than DM $D$. From Eq. (25), we can obtain a condition for the minimum value of $g_{H}$,

$$
g_{H \min }=\frac{2 m_{D}}{\sqrt{v^{2}+v_{\Phi}^{2}+4 v_{\Delta}^{2}}} .
$$

Additionally, we will require that the gauge bosons $Z^{\prime}$ and $Z^{\prime \prime}$ are both heavier than the SM-like $Z$ and that the latter has a mass within its $3 \sigma$ measured value of $91.1876 \pm 0.0021 \mathrm{GeV}$.

To keep heavy fermions above detection limits, we will consider their masses to be no less than $1.5 \mathrm{TeV}$ from the searches of supersymmetric colored particles quoted in the Particle Data Group [73] or $1.2 \times m_{D}$ from coannihilation consideration. In addition, we want to keep the new Yukawa couplings, related to the new heavy fermion masses generically by ${ }^{5} m_{f^{H}}=y_{f^{H}} v_{\Phi} / \sqrt{2}$, to be reasonably small in order to minimize their effects on perturbative unitarity and renormalization group running effects. Therefore, we use the following formula to determine the appropriate Yukawa couplings for each point in our scan:

$$
y_{f^{H}}=\max \left[\frac{1.5 \mathrm{TeV}}{v_{\Phi} / \sqrt{2}}, \min \left(\frac{1.2 m_{D}}{v_{\Phi} / \sqrt{2}}, 1\right)\right] .
$$

Given the size of $v_{\Phi}$ and the fact that $m_{D}$ has to be the lightest $\mathcal{Z}_{2}$-odd particle, we expect that Eq. (41) to easily remain below 1 for all our parameter space. Thus, in this

\footnotetext{
${ }^{4}$ For the Goldstone-like DM scenario, the scan range of $v_{\Phi}$ is fine-tuned to a smaller range from 20 to $28 \mathrm{TeV}$ in order to realize this scenario.

${ }^{5}$ We note that while the Yukawa couplings among the SM fermions and the neutral Higgses maintain flavor diagonal in G2HDM, the new Yukawa couplings are in general not. For simplicity, we have set the unitary mixing matrices among different flavors of heavy and SM fermions in the new Yukawa couplings with the $\mathcal{Z}_{2}$-odd scalars to be the identity matrix.
}

setup, one expects most coannihilation contributions come from other $\mathcal{Z}_{2}$-odd particles such as $\tilde{\Delta}, H^{ \pm}$, and $W^{\prime}$.

From these two steps, we collect $\sim 5$ million points that include numerical values for model parameters, and results from scalar and gauge bosons masses, and the elements of three mixing matrices $\mathcal{O}, \mathcal{O}^{D}$, and $\mathcal{O}^{G}$. We pass these numbers to micromEGAs [65] to calculate relic density, DM-nucleon cross section, and annihilation cross section at present time. Finally, the annihilation cross section and annihilation channels composition are passed to LikeDM [68] for the calculation of indirect detection likelihood.

Due to the notably less abundant nature of doubletlike solutions compared to the other two compositions of DM, a scanning dedicated to find doubletlike solutions was made. For a $M_{H \Delta} \ll v_{\Delta}$, we can make the $(2,2)$ entry in the mass matrix in Eq. (21) smaller than the $(3,3)$ one with the condition

$$
\lambda_{H \Phi}^{\prime}<\frac{M_{\Phi \Delta}}{2 v_{\Delta}} .
$$

Applying this condition increases the abundance of solutions where the lightest complex scalar composition is dominated by $H_{2}^{0 *}$. This explains the far more limited scan range for the parameter $\lambda_{H \Phi}^{\prime}$ for the doubletlike DM case. The complete set of parameters scanned and their ranges can be found in Table III.

Note that in Table III, the different ranges for $M_{H \Delta}, M_{\Phi \Delta}$, $v_{\Delta}$, and $v_{\Phi}$ are selected for the three cases so that we can easily find the corresponding DM composition. In particular, the very different and smaller fine-tuned ranges of $v_{\Delta}$ and $v_{\Phi}$ in the Goldstone-like column are due to this composition being present for $v_{\Delta} / v_{\Phi} \approx 0.8$ but limited by EWPT to be less than $\sim 0.9$, as demonstrated earlier near the end of Sec. III.

Before embarking upon the numerical results, we make some comments on the Sommerfeld enhancement $[74,75]$ in the DM annihilation cross section for indirect detection which may be important whenever $m_{\chi} / m_{\mathcal{M}}>4 \pi / g^{2}$. Here $m_{\chi}$ and $m_{\mathcal{M}}$ denote the masses of the fermionic DM $\chi$ and vector mediator $\mathcal{M}$, respectively, and $g$ is the gauge coupling. In G2HDM, the DM is a complex scalar $D$ and the mediators can be either the Higgses $h_{i}$ or neutral gauge bosons $Z_{i}$. Since all their masses are quite massive and not too distinct from each other, we do not expect significant Sommerfeld enhancement in G2HDM. Certainly, a more decent study is necessary in order to provide a definite answer. Furthermore, we will see in our analysis below that the direct detection limit from XENON1T will provide more stringent constraints than the current indirect detection results from Fermi-LAT. We will ignore such effects in the present analysis. 
TABLE III. Parameter ranges used in the scans mentioned in the text. $M_{X}$ is fixed at $2 \mathrm{TeV}$ in this work and $M_{Y}$ is set to be zero throughout the scan.

\begin{tabular}{lccc}
\hline \hline Parameter & Doubletlike & Tripletlike & Goldstone-like \\
\hline$\lambda_{H}$ & {$[0.12,2.75]$} & {$[0.12,2.75]$} & {$[0.12,2.75]$} \\
$\lambda_{\Phi}$ & {$\left[10^{-4}, 4.25\right]$} & {$\left[10^{-4}, 4.25\right]$} & {$\left[10^{-4}, 4.25\right]$} \\
$\lambda_{\Delta}$ & {$\left[10^{-4}, 5.2\right]$} & {$\left[10^{-4}, 5.2\right]$} & {$\left[10^{-4}, 5.2\right]$} \\
$\lambda_{H \Phi}$ & {$[-6.2,4.3]$} & {$[-6.2,4.3]$} & {$[-6.2,4.3]$} \\
$\lambda_{H \Delta}$ & {$[-4.0,10.5]$} & {$[-4.0,10.5]$} & {$[-4.0,10.5]$} \\
$\lambda_{\Phi \Delta}$ & {$[-5.5,15.0]$} & {$[-5.5,15.0]$} & {$[-5.5,15.0]$} \\
$\lambda_{H \Phi}^{\prime}$ & {$[-1.0,18.0]$} & {$[-1.0,18.0]$} & {$[-1.0,18.0]$} \\
$\lambda_{H}^{\prime}$ & {$[-8 \sqrt{2} \pi, 8 \sqrt{2} \pi]$} & {$[-8 \sqrt{2} \pi, 8 \sqrt{2} \pi]$} & {$[-8 \sqrt{2} \pi, 8 \sqrt{2} \pi]$} \\
$g_{H}$ & {$[S e \mathrm{etext}, 0.1]$} & {$[$ See text, 0.1$]$} & {$[$ See text, 0.1$]$} \\
$g_{X}$ & {$\left[10^{-8}, 1.0\right]$} & {$\left[10^{-8}, 1.0\right]$} & {$\left[10^{-8}, 1.0\right]$} \\
$M_{H \Delta} / \mathrm{GeV}$ & {$[0.0,15000]$} & {$[0.0,5000.0]$} & {$[0.0,5000.0]$} \\
$M_{\Phi \Delta} / \mathrm{GeV}$ & {$[0.0,5.0]$} & {$[-50.0,50.0]$} & {$[0.0,700]$} \\
$v_{\Delta} / \mathrm{TeV}$ & {$[0.5,2.0]$} & {$[0.5,20.0]$} & {$[14.0,20.0]$} \\
$v_{\Phi} / \mathrm{TeV}$ & {$[20,100]$} & {$[20,100]$} & {$[20,28.0]$} \\
\hline \hline
\end{tabular}

\section{B. Results}

To ease the discussion of our numerical results, it is useful to divide the DM mass range into several regions as follows:

(i) Light DM mass region where annihilation final states of $c \bar{c}$ and $\tau^{+} \tau^{-}$are opened

(ii) The resonance region where DM mass is close to SM $Z$ or Higgs resonance.

(iii) The intermediate DM mass from Higgs resonance $m_{h_{1}} / 2$ to $\sim 500 \mathrm{GeV}$ where DM mainly annihilates to $W^{+} W^{-}$and $Z Z$.

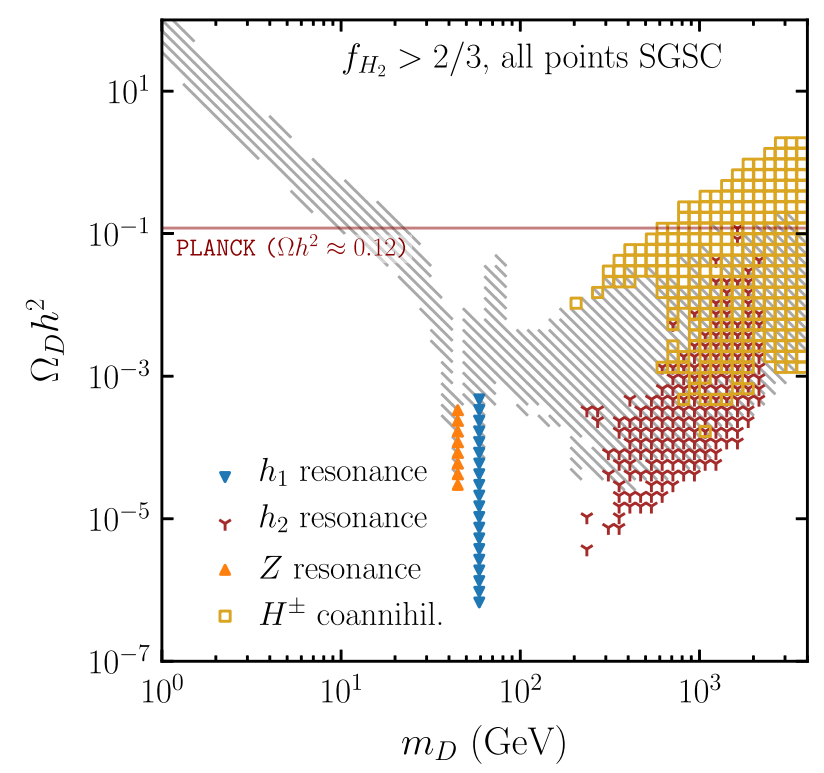

(iv) The heavy DM mass larger than $500 \mathrm{GeV}$.

\section{Inert doubletlike DM}

The doubletlike DM in G2HDM is similar to the IHDM case in the limit where the scalar $(S)$ and pseudoscalar $(P)$ components in $H_{2}^{0}$ are mass degenerate. We show the scatter plot for the relic density dependence on the DM mass in the left panel of Fig. 4. Similar to Refs. [13,76], there are several different annihilation mechanisms governing different DM mass regions. However, the observed

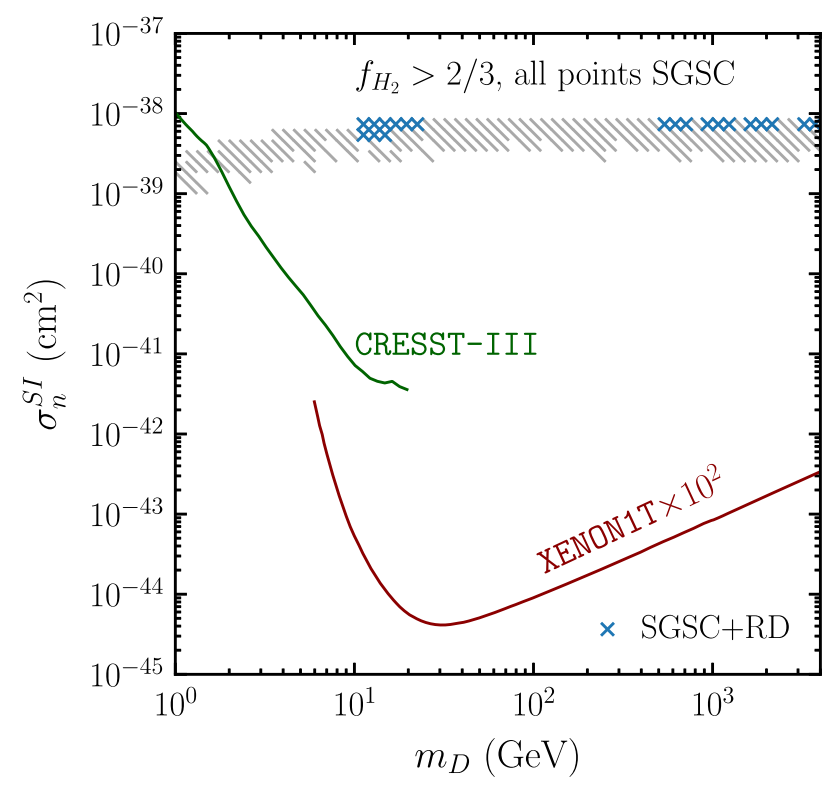

FIG. 4. Doubletlike DM SGSC allowed regions projected on $\left(m_{D}, \Omega_{D} h^{2}\right)$ (left) and $\left(m_{D}, \sigma_{n}^{S I}\right)$ (right) planes. The gray area in the left panel has no coannihilation or resonance. The gray area in the right panel is excluded by PLANCK data at $2 \sigma$. 
relic abundance $\Omega_{D} h^{2} \approx 0.1$ only occurs at around $m_{D} \sim$ $10 \mathrm{GeV}$ and $m_{D}>500 \mathrm{GeV}$.

In the following, we discuss in more detail the DM annihilations for this inert doubletlike DM case in the four DM mass regions (i)-(iv) consecutively.

(i) First, the DM masses that lie between 1 and $10 \mathrm{GeV}$ whose major contributions of the DM annihilation cross section are given by $D D^{*} \rightarrow c \bar{c}$ and $\tau^{+} \tau^{-}$via $s$-channel SM Higgs exchange. Despite of the small $c$ and $\tau$ Yukawa couplings, the cross section can be slightly enhanced by the relatively big $D D^{*} h_{i}$ coupling, as given in Eq. (A4). Thanks to large values for $\lambda_{H \Phi} v_{\phi}$ and $\lambda_{H \Delta} v_{\Delta}$. However, the total cross section is too small to bring the relic density in this mass range closer to the PLANCK measurement. Due to the opening of the $D D^{*} \rightarrow b \bar{b}$ channel and its larger Yukawa coupling, the correct relic density can be obtained for DM masses between 11 and $20 \mathrm{GeV}$.

(ii) When DM mass is around half the $Z$ mass, the $Z$ exchange diagram becomes very efficient and the enhancement in resonant annihilation brings the relic density well below 0.12 of the PLANCK measurement. We note that $D D^{*} Z_{i}$ couplings are unique in G2HDM due to the nature of complex scalar. In IHDM, the DM can be either the real or imaginary parts of $H_{2}^{0}=S+i P$, in which case neither the $S S Z$ nor $P P Z$ coupling is present. Similarly, it also happens for DM mass at around half the SM Higgs mass, where again, enhanced annihilation rate through Higgs exchange brings the relic density even lower.

(iii) If DM mass is increased above half the SM Higgs mass $\left(m_{h_{1}} / 2<m_{D}<500 \mathrm{GeV}\right)$, the Higgs resonance is no longer efficient. However, once the gauge boson final state, especially $W^{+} W^{-}$, opens $\left(m_{D}>m_{W}\right)$, the total cross section is governed by the process $D D^{*} \rightarrow W^{+} W^{-}$. The relevant diagrams for this process are the four-point interaction $D D^{*} W^{+} W^{-}, s$ channel mediated by each $h_{i}$ and each $Z_{i}$ gauge bosons, and $t$ and $u$ channels with charged Higgs mediator. The dominant channel is the $s$ channel through lightest Higgs $h_{1}$ (not efficient but non-negligible) and second lightest Higgs $h_{2}$ exchange. The third Higgs $h_{3}$ is too heavy and not relevant. Thus, the annihilation cross section is determined by the $D D^{*} h_{1}$ and $D D^{*} h_{2}$ couplings. These two couplings have terms proportional to each of the three VEVs [see Eq. (A4)], which are usually too large in order to have enough relic density at the DM mass region below $500 \mathrm{GeV}$. As a result, the observed relic density (within $2 \sigma$ region) cannot be satisfied in this regime as one can see in the left panel of Fig. 4. The other three final states opening in this intermediate mass range are $Z Z, h_{1} h_{1}$, and $t \bar{t}$, which are all subdominant compared with the $W^{+} W^{-}$final state.

(iv) Finally, in the heavy mass region $\left(m_{D}>500 \mathrm{GeV}\right)$, the dominant final states are from the longitudinal components of the gauge bosons, namely $W_{L}^{+} W_{L}^{-}$ and $Z_{L} Z_{L}$. For $Z_{L} Z_{L}$ final state, there is an exact cancellation between the four-point contact interaction diagram and the $t$ and $u$ channels of $D$ exchange diagrams. The sum of these three diagrams is proportional to $\left(s+t+u-2 m_{D}^{2}-2 m_{Z}^{2}\right)$ and hence vanishes identically due to kinematical constraint. Thus, the remaining diagrams for $D D^{*} \rightarrow$ $Z_{L} Z_{L}$ are given by the $s$-channel $h_{i}$ exchange which lead to $S$-wave total cross section in the nonrelativistic limit. There is a similar cancellation between the four-point contact interaction diagram and the $t$ channel charged Higgs exchange diagram for the $W_{L}^{+} W_{L}^{-}$final state. The sum of the amplitudes from these two contributions is given by

$$
\begin{aligned}
& \mathcal{A}_{(4-\mathrm{pt}+\text { Charged Higgs })} \\
& \quad \approx \frac{e^{2}\left(\mathcal{O}_{22}^{D}\right)^{2}}{2 m_{W}^{2} s_{W}^{2}}\left[\frac{\left(s-2 m_{W}^{2}\right)}{2}+\frac{\left(t-m_{D}^{2}\right)^{2}}{\left(t-m_{H^{ \pm}}^{2}\right)}\right],
\end{aligned}
$$

where $t=m_{D}^{2}+m_{W}^{2}-s / 2$. Clearly, when $s$ is sufficiently large such that all masses can be ignored and $t \sim-s / 2$, the above amplitude vanishes. However, one notes that if $D-H^{ \pm}$coannihilation happens for this heavy DM mass region, i.e., when $m_{D} \simeq m_{H^{ \pm}}$, the above amplitude also vanishes. Thus, in the heavy DM mass region, where the $D-$ $H^{ \pm}$coannihilation occurs, the dominant diagrams that contribute to $D D^{*} \rightarrow W_{L}^{+} W_{L}^{-}$are the $h_{i}$ and $Z_{i}$ exchanges which give rise to $S$ - and $P$-wave total cross sections, respectively, in the nonrelativistic limit. We can also conclude that the total cross sections for DM annihilation into both $W_{L}^{+} W_{L}^{-}$and $Z_{L} Z_{L}$ final states in G2HDM are consistent with unitarity [77].

In the right panel of Fig. 4, we show the scatter plot for the spin independent direct detection cross section versus the DM mass. The interactions between DM and nucleons are mediated by $t$-channel $h_{i}$ and $Z_{i}$ boson exchange, with a small contribution from $u$-channel heavy fermion exchange. Due to the $S U(2)_{L} \times U(1)_{Y}$ charge of the inert doublet $\mathrm{H}_{2}$, the doubletlike DM-nucleon cross section is dominated by $Z$ exchange. As one can see in the plot, the doubletlike DM in G2HDM predicts a typical value of the cross section of order $10^{-38} \mathrm{~cm}^{2}$. It can be excluded by XENON1T [63] and CRESST-III [78] down to DM masses above $2 \mathrm{GeV}$. For the points below $2 \mathrm{GeV}$ that survive the CRESST-III constraint, the predicted relic abundance is always higher than the measured PLANCK value. Regarding the ISV effects, we check that $\left|f_{n} / f_{p}\right|$ remains 

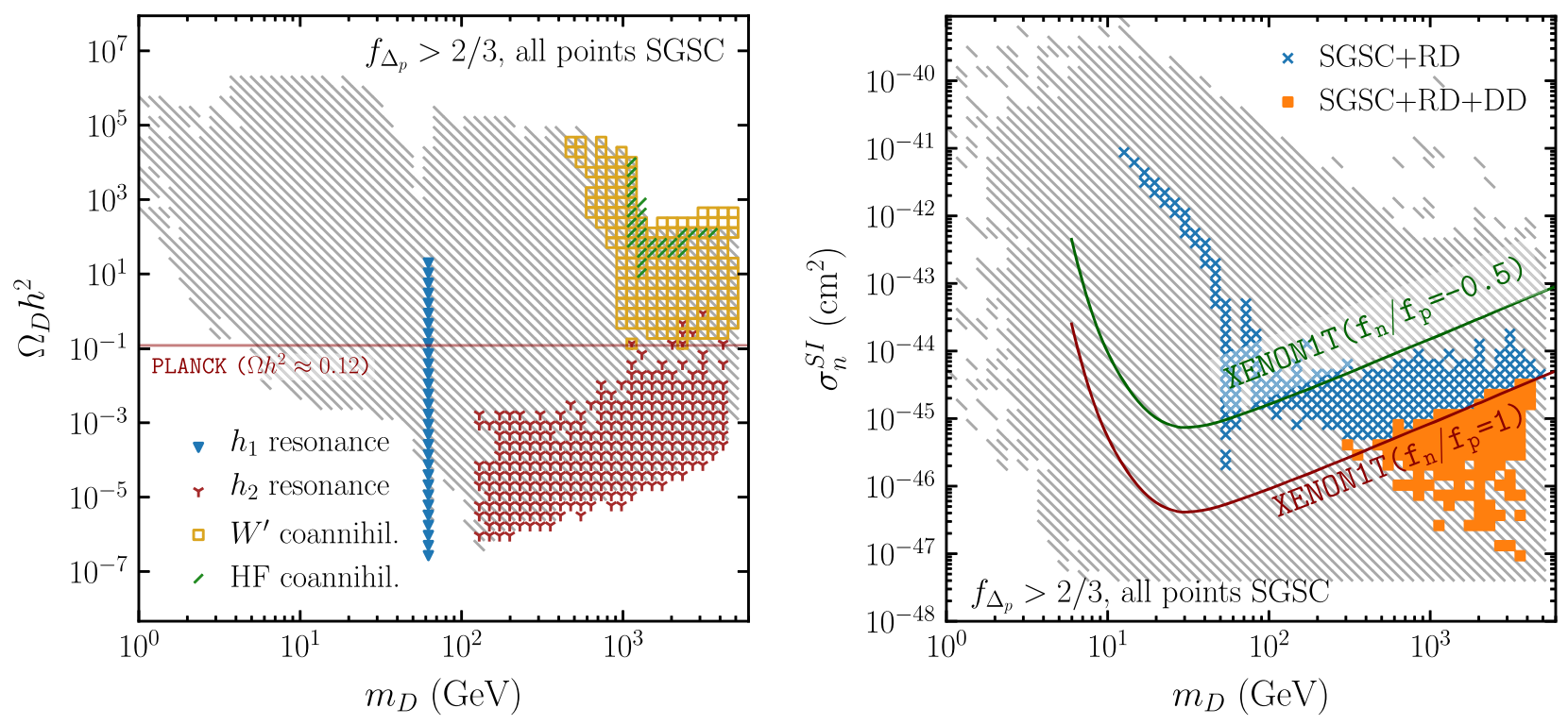

FIG. 5. Tripletlike DM SGSC allowed regions projected on $\left(m_{D}, \Omega_{D} h^{2}\right)$ (left) and $\left(m_{D}, \sigma_{n}^{S I}\right)$ (right) planes. The gray area in the left panel has no coannihilation or resonance. The gray area in the right panel is excluded by PLANCK data at $2 \sigma$. In the right panel, the lower red solid line is the published XENON1T limit with isospin conservation, while the upper green solid line is the same limit but for ISV with $f_{n} / f_{p}=-0.5$. Some orange filled squares are above the published XENON1T limit due to ISV cancellation at nucleus level.

typically 3 orders of magnitude far away from the maximal cancellation value of $f_{n} / f_{p} \approx-0.7$. Therefore, there is no noticeable reduction in the nucleon-level DD cross section.

It is clear from the previous discussion that for the doubletlike DM case there is no surviving parameter space that can remain after the constraints from both PLANCK and XENON1T are taken into account. Therefore, doubletlike DM in G2HDM is completely ruled out by current experiments, at least under the somewhat generic conditions setup in this paper. A study of particular mechanisms or very specific sets of parameters (e.g., a very light mediator region) that may bring down the relic density for light doubletlike DM $(\sim 1 \mathrm{GeV})$ while keeping the prediction of direct detection intact is out of the scope of the present analysis.

\section{2. $S U(2)_{H}$ tripletlike $D M$}

One fundamental difference between tripletlike and doubletlike DM is that now $D$ is dominated by the term $\mathcal{O}_{32}^{D} \Delta_{p}$ in Eq. (29). Therefore, one should expect all the couplings to behave differently from the previous doubletlike case. In particular, the coupling terms that were relevant for doubletlike DM will now be suppressed by a smaller $\mathcal{O}_{22}^{D}$. In Fig. 5, we show the scatter plots for the relic density and spin independent direct detection cross section versus the DM mass $m_{D}$ at the left and right panels, respectively, for the tripletlike case. Similar to the doubletlike case, one can divide the DM mass in different regions for discussions. The opening channels are the same in each region and hence no need to repeat here. However, the dominant channels in each region may be changed due to the differences of the couplings in both DM compositions.

For the relic density, the resulting resonances and coannihilation regions are presented in the left panel of Fig. 5. We found that in the DM mass range below $m_{h_{1}} / 2$ [region (i)], the dominant DM annihilation contribution to the relic abundance comes from $s$-channel Higgses exchange with final states of $\tau^{+} \tau^{-}$and $b \bar{b}$. By looking at the $D D^{*} h_{i}$ coupling in Eq. (A6), it is easy to see that the large value of $v_{\Phi}$ makes annihilation through $h_{2}$ ( $\delta_{3}$-like) comparable with annihilation through $h_{1}$ (SM-like) while the heavier $h_{3}\left(\phi_{2}\right.$-like) contribution remains subleading. As expected, the lowest relic density happens at the Higgs resonance region $m_{D} \approx m_{h_{1}} / 2$, combining with the large $D D^{*} h_{1}$ coupling from the first term of Eq. (A6). Of course, one can always decrease the values of $v_{\Phi}$ or $v_{\Delta}$ to reduce the coupling size for larger relic abundance, but this is not particularly interesting for a thermal DM scenario. As DM becomes heavier, other resonance turns on. For $m_{D}>100 \mathrm{GeV}, D$ is massive enough to have points where $2 m_{D} \approx m_{h_{2}}$ and some points resulting in resonant annihilation through $s$-channel $h_{2}$ exchange. In contrast to the doubletlike scenario, it is possible for the tripletlike DM to have a very wide range of relic density values, given the several different possible combinations for the $D D^{*} h_{i}$ coupling in Eq. (A6). Unlike the doubletlike case, for the tripletlike DM case in region (ii), the reduction of the relic density due to the $Z$-resonance enhancement in the annihilation cross section is absent because the triplet $\Delta_{H}$ is a SM singlet, meaning that the interaction between DM and 

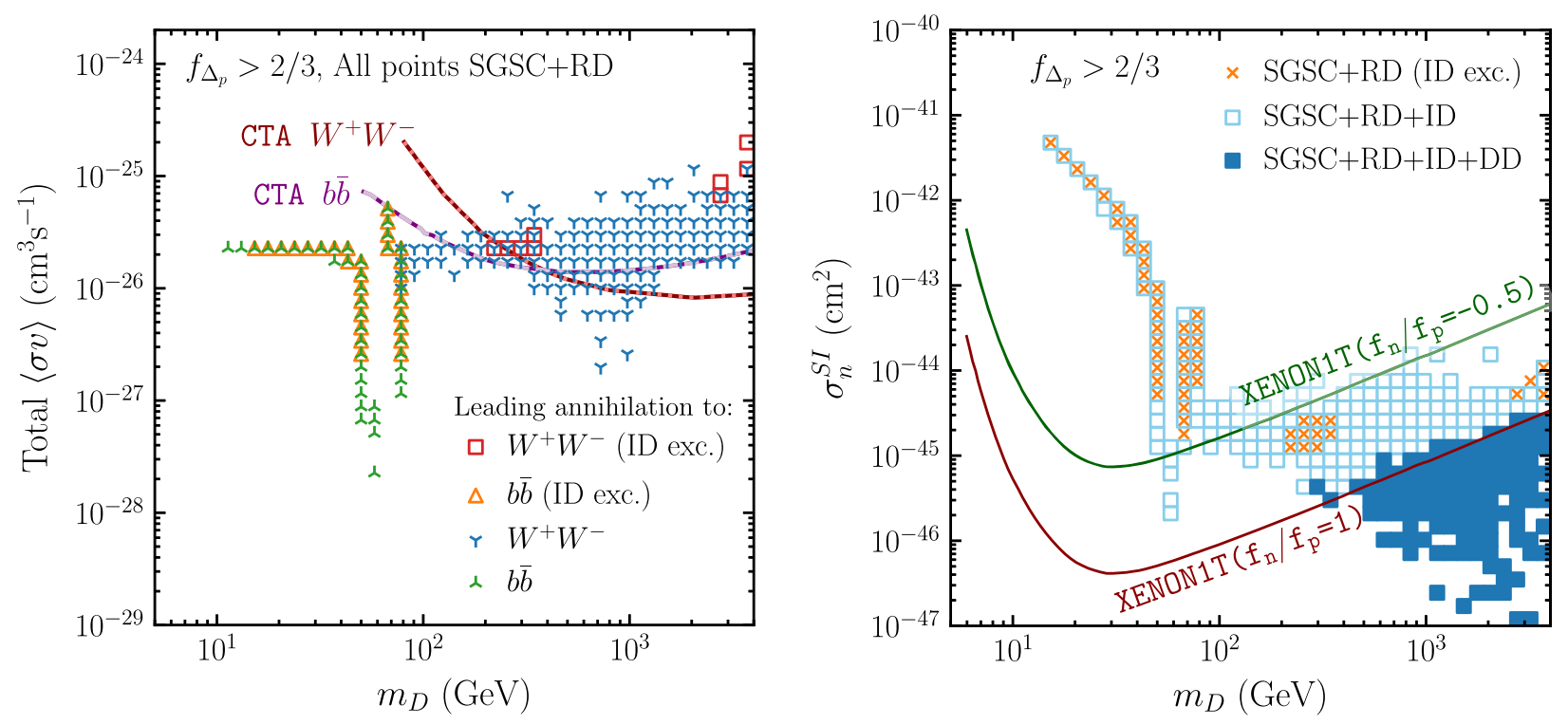

FIG. 6. The present time total annihilation cross section according to dominant annihilation channel (left) and DM-neutron elastic scattering cross section (right) for $f_{\Delta_{p}}>2 / 3$ in the tripletlike DM case versus the DM mass $m_{D}$. Two-dimensional $2 \sigma$ criteria of the ID constraints is $\Delta \chi^{2}=5.99$ based on Fermi dSphs gamma-ray flux data. Future Cherenkov Telescope Array (CTA) measurements may help constrain regions with DM masses above $\mathcal{O}\left(10^{2}\right) \mathrm{GeV}$ as shown in the left panel. In the right panel, the lower red solid line is the published XENON1T limit with isospin conservation, while the upper green solid line is the same limit but for ISV with $f_{n} / f_{p}=-0.5$. Some blue filled squares are above the published XENON1T limit due to ISV cancellation at nucleus level.

the $\mathrm{SM} Z$ is suppressed by product of small mixing elements like $\left(\mathcal{O}_{32}^{D}\right)^{2} \mathcal{O}_{21}^{G}$ according to Eq. (A5).

In region (iii) where $m_{h_{1}} / 2<m_{D}<500 \mathrm{GeV}$, the relic density reduction mechanism is similar to the doubletlike DM case discussed above. The annihilation cross section is highly dominated by $W^{+} W^{-}$(more than $\sim 50 \%$ ), $h_{1} h_{1}$ ( 25\%), and $Z Z(\sim 20 \%)$ final states. The main contribution to the $W^{+} W^{-}$final state comes from $S$ wave given by $h_{i}$ exchange, while the $P$-wave contribution is suppressed and originated from neutral gauge bosons mediator exchange. The $S$-wave annihilation cross section is controlled by the $D D^{*} h_{1}$ and $D D^{*} h_{2}$ couplings, as can be seen in Eq. (A6). The contribution from $h_{3}$ exchange is negligible because of its heavy mass.

In region (iv) where $m_{D}>500 \mathrm{GeV}, \mathrm{DM}$ annihilates into $W_{L}^{+} W_{L}^{-}$predominantly while other channels are subdominant, similar to the doubletlike DM case. There is no need to elaborate further here.

Generally speaking, the charged Higgs $H^{ \pm}$contribution here can be omitted since it is more than twice heavier than the DM $D$. Differently from the doubletlike case, there is no coannihilation between $H^{ \pm}$and $D$ in the tripletlike DM case. Next, the coannihilation between DM and $\tilde{\Delta}$ is absent as well because the $\tilde{\Delta}$ is also much heavier than $D$ due to the choice of larger $v_{\Delta}$ to make the $(3,3)$ entry of Eq. (21) smaller. Therefore, the only possible efficient coannihilation is between $\mathrm{DM}$ and $W^{\prime}$ for $\mathrm{DM}$ mass above $400 \mathrm{GeV}$ (orange boxes at the left panel of Fig. 5). This coannihilation is only important for relic density above 0.12 , where some $D D^{*}$ annihilation channels may be insufficient because their couplings to $h_{i}$ and $Z_{j}$ may be suppressed. A small region with heavy fermion coannihilation happens for $m_{D}>$ $1 \mathrm{TeV}$ with relic density above 10 (green shaded points in the left panel of Fig. 5). This is close to the maximal relic density in our scan for that mass range. This indicates that heavy fermion coannihilation is important only when the other annihilation channels are strongly suppressed.

Regarding direct detection, due to the $D D^{*} Z$ coupling suppression by mixings in this tripletlike case, the elastic DM-nucleon scattering spin independent cross section mediated by $h_{i}$ and the extra neutral gauge bosons $Z^{\prime}$ and $Z^{\prime \prime}$ bosons may be relevant. We confirm that the dominant contribution to the spin independent cross section is given by $h_{1}$ and the next dominant contributions are $Z$ and $Z^{\prime}$, while $h_{2}, h_{3}$, and $Z^{\prime \prime}$ are always subdominant. The contributions mediated by heavy fermions are negligible due to suppression by their masses in the propagators.

In the right panel of Fig. 5, we can see that for $m_{D} \gtrsim$ $300 \mathrm{GeV}$ it is possible to find a region that agrees with relic density constraint from PLANCK at $2 \sigma$ and remains below the published XENON1T limit at the neutron with $f_{n} / f_{p}=1$. Note that some of the allowed points (orange squares) are above this XENON1T limit. This is due to mild ISV cancellation that brings such points below the XENON1T limit at nucleus level, as given by Eq. (33). For comparison, the XENON1T limit at the neutron level with ISV of $f_{n} / f_{p}=-0.5$ is also shown.

The constraint of indirect detection from Fermi-LAT's gamma-ray observation imposed on the tripletlike DM is shown in Fig. 6. The left panel presents the DM 

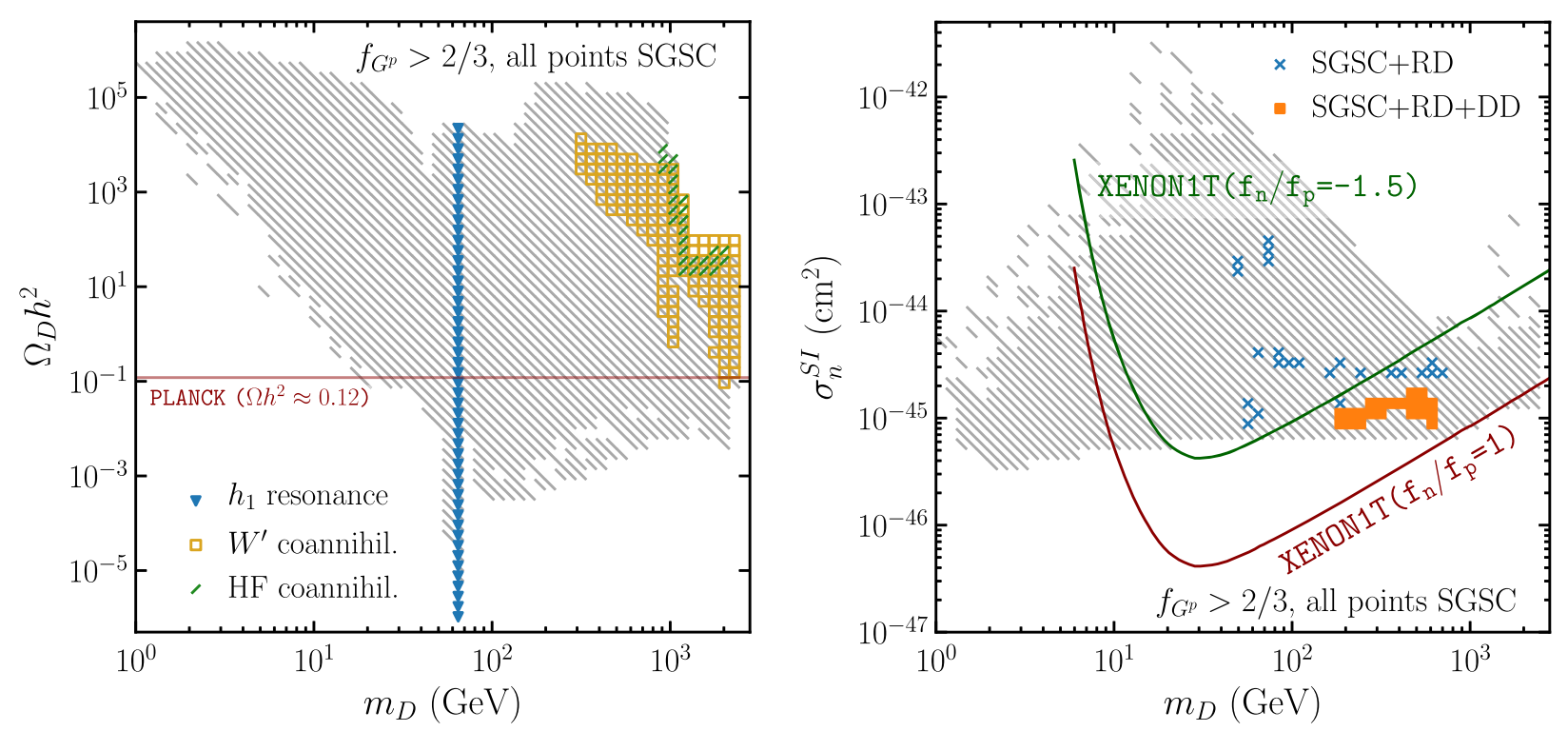

FIG. 7. Goldstone-like DM SGSC allowed regions projected on $\left(m_{D}, \Omega_{D} h^{2}\right)$ (left) and $\left(m_{D}, \sigma_{n}^{S I}\right)$ (right) planes. The gray area in the left panel has no coannihilation or resonance. The gray area on the right is excluded by PLANCK data at $2 \sigma$. In the right panel, the lower red solid line is the published XENON1T limit with isospin conservation, while the upper green solid line is the same limit but for ISV with $f_{n} / f_{p}=-1.5$. The small region of orange filled squares above the published XENON1T limit presents ISV cancellation at nucleus level.

annihilation cross section dependence ${ }^{6}$ on the DM mass at the present Universe with SGSC + RD. Results with dominant annihilation channels of either $b \bar{b}$ or $W^{+} W^{-}$ are indicated explicitly. One can see that DM with $m_{D} \lesssim$ $90 \mathrm{GeV}$ mainly annihilates to $b \bar{b}$. At the region near the $Z$ or $h_{1}$ resonance, the corresponding cross section at the present Universe drops while satisfying the relic density. This is a typical feature of the resonance region because the DM relative velocity at the early Universe is much larger than the value at the present one. In order to cancel a large cross section caused by the resonance at the early Universe, a small coupling of $D D^{*} Z$ or $D D^{*} h_{1}$ is required to make $\langle\sigma v\rangle$ at the early Universe comes close to the canonical value of $10^{-26} \mathrm{~cm}^{3} \cdot \mathrm{s}^{-1}$. However, when the Universe temperature drops, the resonance cannot be maintained by the kinetic energy of DM at the present day. At this time, the cross section becomes smaller and is hard to be observed by Fermi-LAT.

Once DM mass is heavier than $W^{ \pm}$boson mass, the final state $W^{+} W^{-}$starts dominating the annihilation cross section rapidly. Note that the current ID sensitivity can only apply strongly for the DM mass located between $10 \mathrm{GeV}$ and few hundred GeV. However, the future CTA sensitivity [79] might reach the $\mathrm{TeV}$ region of $m_{D}$ and further constrain our parameter space, as show in the left panel of Fig. 6.

\footnotetext{
${ }^{6}$ Note that to apply Fermi-LAT constraints we use photon flux as calculated with Eq. (36). The annihilation channels displayed in Fig. 6 are only leading channels that may not be significantly above other channels.
}

In the right panel of Fig. 6, we display the exclusion from ID projected on the plane of DM-neutron spin independent cross section $\sigma_{n}^{\text {SI }}$ versus $m_{D}$. We can see that all the ID excluded points sit above the limit set by XENON1T. The exclusion limits are given by recent XENON1T data (blue unfilled squares) and Fermi gamma-ray constraints (orange crosses). One can see the XENON1T exclusion power is much stronger than Fermi gamma-ray exclusion.

\section{3. $S U(2)_{H}$ Goldstone bosonlike DM}

In this case, as shown at the end of Sec. III (see Fig. 1), the Goldstone-like DM $D$ will be a mixture dominated by $G_{H}^{p}$ with an important component coming from $\Delta_{p}$, while the $H_{2}^{0 *}$ component remains suppressed. In the left panel of Fig. 7, for the DM mass regions (i)-(iii), the dominant channels for DM annihilation in the relic abundance calculation are similar to the tripletlike DM case. In heavy mass region (iv), the cross section is again dominated by the $W_{L}^{+} W_{L}^{-}$final state which contributes $\sim 50 \%$, while the transverse component is negligible. The main difference between Goldstone-like and tripletlike DM can be understood by their corresponding dominant couplings. For tripletlike DM, the dominant couplings are given by Eqs. (A5) and (A6) which are proportional to the $\left(\mathcal{O}_{32}^{D}\right)^{2}$ characterizing the corresponding $\Delta_{p}$ component. Similarly, one expects that the Goldstone-like DM receives its dominant couplings purely via Eqs. (A7) and (A8). However, this is not the case for Goldstone-like DM. There is also an important contribution coming from the $\Delta_{p}$ part in the relevant couplings. Thus, one needs to 

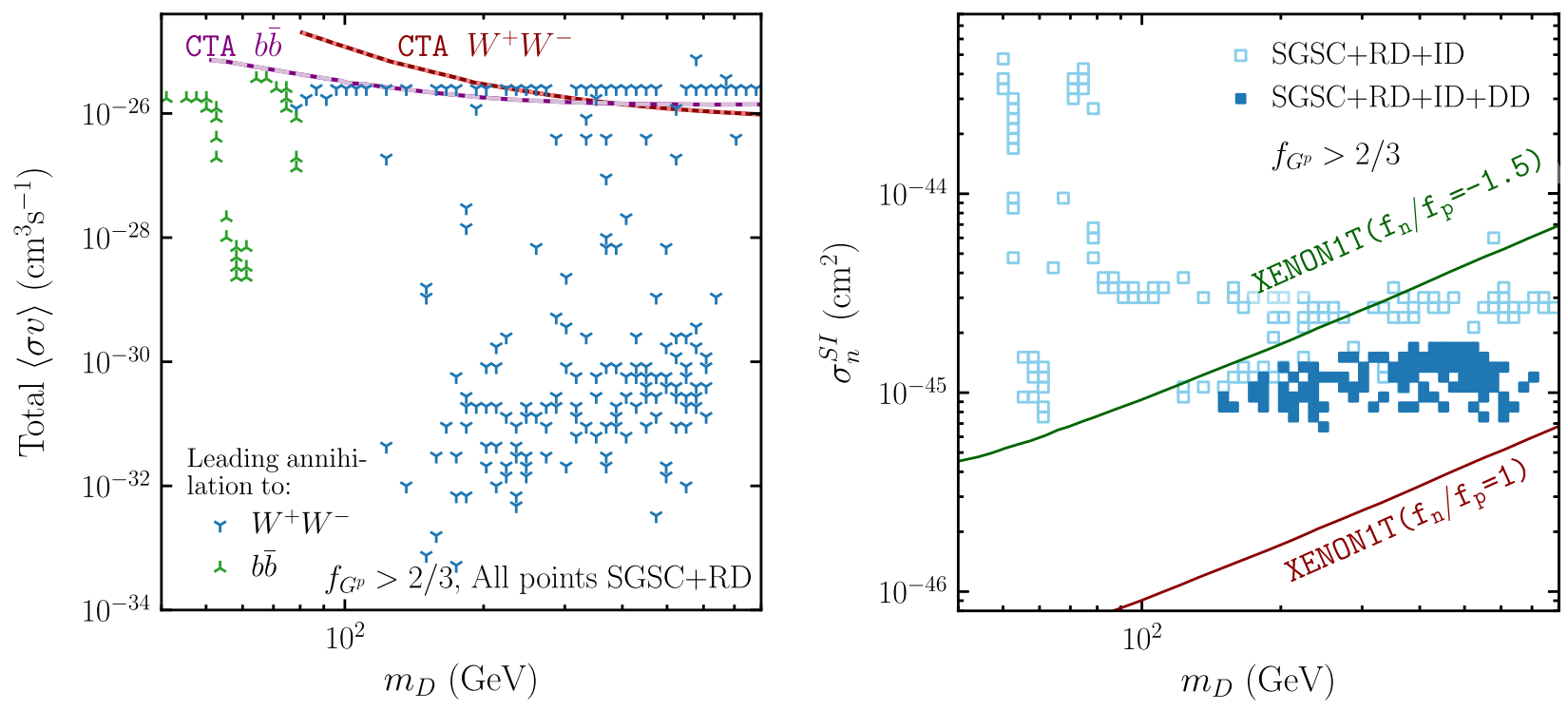

FIG. 8. The present time total annihilation cross section by dominant annihilation channels (left) and DM-neutron elastic scattering cross section (right) for $f_{G^{p}}>2 / 3$ in the Goldstone-like DM case versus the DM mass $m_{D}$. Two-dimensional $2 \sigma$ criteria of the ID constraints is $\Delta \chi^{2}=5.99$ based on Fermi dSphs gamma-ray flux data. Future CTA measurements may help constrain regions with DM masses above $\mathcal{O}\left(10^{2}\right) \mathrm{GeV}$ as shown in the left panel. In the right panel, the lower red solid line is the published XENON1T limit with isospin conservation, while the upper green solid line is the same limit but for ISV with $f_{n} / f_{p}=-1.5$. Some blue filled squares are above the published XENON1T limit due to ISV cancellation at nucleus level.

include not only the couplings proportional to $\left(\mathcal{O}_{12}^{D}\right)^{2}$ but also the ones proportional to $\left(\mathcal{O}_{32}^{D}\right)^{2}$. The effect of $\Delta_{p}$ component in this case is reducing the $D D^{*} h_{1}$ and $D D^{*} h_{2}$ couplings while enhancing the $D D^{*} Z$ and $D D^{*} Z^{\prime}$ couplings. As a consequence, the dominant DM annihilation channel $W_{L}^{+} W_{L}^{-}$will be dominated by $P$-wave component originated from the $Z^{\prime}$ exchange, while the $S$-wave part coming from the $h_{1}$ and $h_{2}$ mediators is subdominant. The next important contribution is given by the $Z^{\prime} Z^{\prime}$ final state. The $Z^{\prime} Z^{\prime}$ final state occurs via four-point contact interaction, $t$ and $u$ channels of $D$ exchange and $s$ channel of neutral Higgses exchange. The presence of the $\Delta_{p}$ component in the Goldstone-like DM further enhances the $D D^{*} Z^{\prime} Z^{\prime}$ coupling resulting in the appearance of the new important final state $Z^{\prime} Z^{\prime}$ in the heavy mass region (iv).

Coannihilation in this case is very similar to the tripletlike DM case. The most relevant coannihilations happen with $W^{\prime}$ and heavy fermions for large masses and large relic density. Coannihilation with $W^{\prime}$ only presents when the $\mathrm{DM}$ mass gets close to $300 \mathrm{GeV}$ and its relic density is mostly above the PLANCK measurement. As the tripletlike DM case, the usual $D D^{*}$ annihilation channels become smaller leaving more way for coannihilations that, otherwise, would be negligible. For the case of heavy fermions, coannihilation happens for DM masses above $1 \mathrm{TeV}$ and mostly for the upper bound of relic density, where $D D^{*}$ coannihilation is even more suppressed than for the $W^{\prime}$ case.

In the right panel of Fig. 7, we show the scatter plot for the DM-neutron cross section dependence on the DM mass.
The dominant contribution comes from $h_{1}$ exchange with the next dominant ones given by the exchange of $Z$ and $Z^{\prime}$ bosons. The bottom part of the gray region in Fig. 7 comes mostly from interactions mediated by the $Z$ and $Z^{\prime}$ gauge bosons and is limited from below by our lower limit for the scan range of $g_{H}$ determined by Eq. (40). The interference between $h_{1}, Z$, and $Z^{\prime}$ exchange makes the spin independent cross section varies in a wide range. The orange points located between $200 \mathrm{GeV} \leq m_{D} \leq 600 \mathrm{GeV}$ satisfy the observed relic density while escaping the current bound on direct detection given by XENON1T experiment. The dominant contribution for these points is given by the gauge bosons exchange $Z$ and $Z^{\prime}$. Due to the fine-tuning parameter space for the Goldstone-like DM mentioned earlier, only the ratio of $f_{n} / f_{p}=-1.86$ has enough ISV cancellation to satisfy the published XENON1T limit assuming isospin conservation. For comparison, the XENON1T limit with ISV of $f_{n} / f_{p}=-1.5$ is also shown.

In the ID side, there is no relevant constraining for this Goldstone bosonlike case. Because of $P$-wave suppression of the $Z$ and $Z^{\prime}$ exchange in the dominant channels of $b \bar{b}$ and $W^{+} W^{-}$, most of the points in agreement with the relic density measurement from PLANCK have a very low annihilation cross section at the present time and are far beyond the reach of current experiments of indirect detection, as can be seen clearly in the zoomed in region on the $\left(\langle\sigma v\rangle, m_{D}\right)$ plane at the left panel in Fig. 8 allowed by the SGSC + RD. For DM masses below $100 \mathrm{GeV}$, the annihilation is dominated by $b \bar{b}$ final state with $90 \%$ of the total cross section in average. For DM mass above the mass 


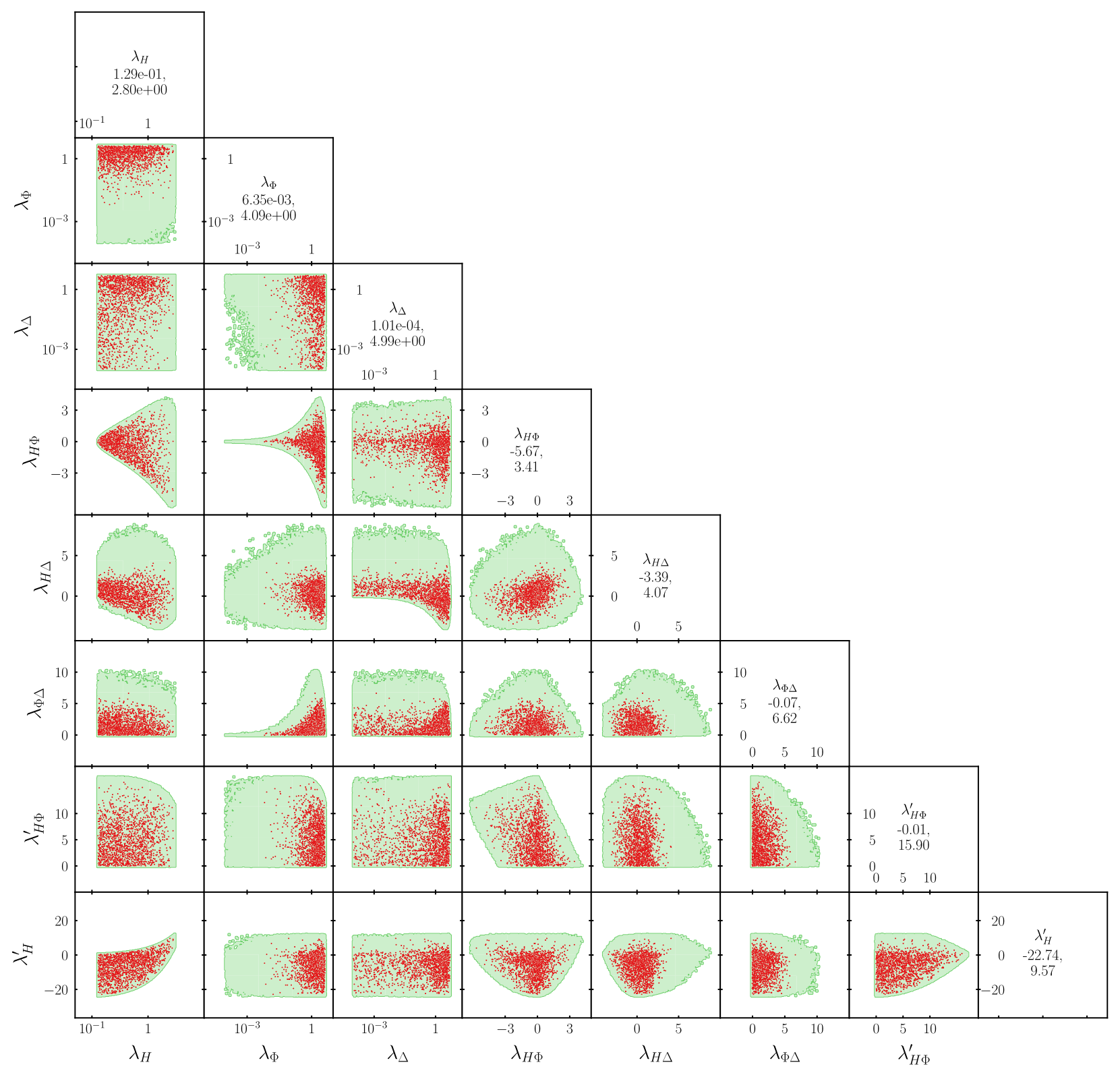

FIG. 9. A summary plot for the scalar potential parameter space allowed by the SGSC constraints (green region) and SGSC+RD+DD constraints (red scatter points) for the tripletlike DM. The numbers written in the first block of each column are the one-dimensional allowed range of the parameter denoted in horizontal axis after the SGSC + RD + DD cut.

of the $W^{ \pm}$, the $W^{+} W^{-}$final state dominates completely with an average of $50 \%$ of the total cross section. Unlike tripletlike DM, ID alone does not further constrain the points allowed by PLANCK. The right panel of Fig. 8 shows the zoomed in region of points on the $\left(m_{D}, \sigma_{n}^{S I}\right)$ plane allowed by the SGSC $+\mathrm{RD}+\mathrm{ID}$ and $\mathrm{SGSC}+$ $\mathrm{RD}+\mathrm{ID}+\mathrm{DD}$. As mentioned before, ISV effect $\left(f_{n} / f_{p} \approx\right.$ $-1.86)$ reduces the sensitivity of the XENON1T result and some points pass all the constraints SGSC+RD+ID+DD even though they are above the direct detection limit at nucleon level. Note that there are no points satisfying SGSC + RD + ID + DD beyond $m_{D} \sim 1 \mathrm{TeV}$ in this Goldstone-like case.

\section{Constraining parameter space in G2HDM}

From previous sections, we have learned that the doubletlike DM scenario cannot fulfill the DM constraints and that the Goldstone-like DM requires some fine-tuning in the parameter space and to escape the XENON1T limit a particular value of $f_{n} / f_{p} \approx-1.86$ is required. Therefore, we will be focusing on discussing the allowed G2HDM parameter space based on the tripletlike DM.

In Fig. 9, we present the allowed regions of the quartic couplings from the SGSC constraints (green region) and SGSC + RD + DD constraints (red scatter points). Comparing the green regions with the red scatter points in Fig. 9, one can easily obtain the following results: 


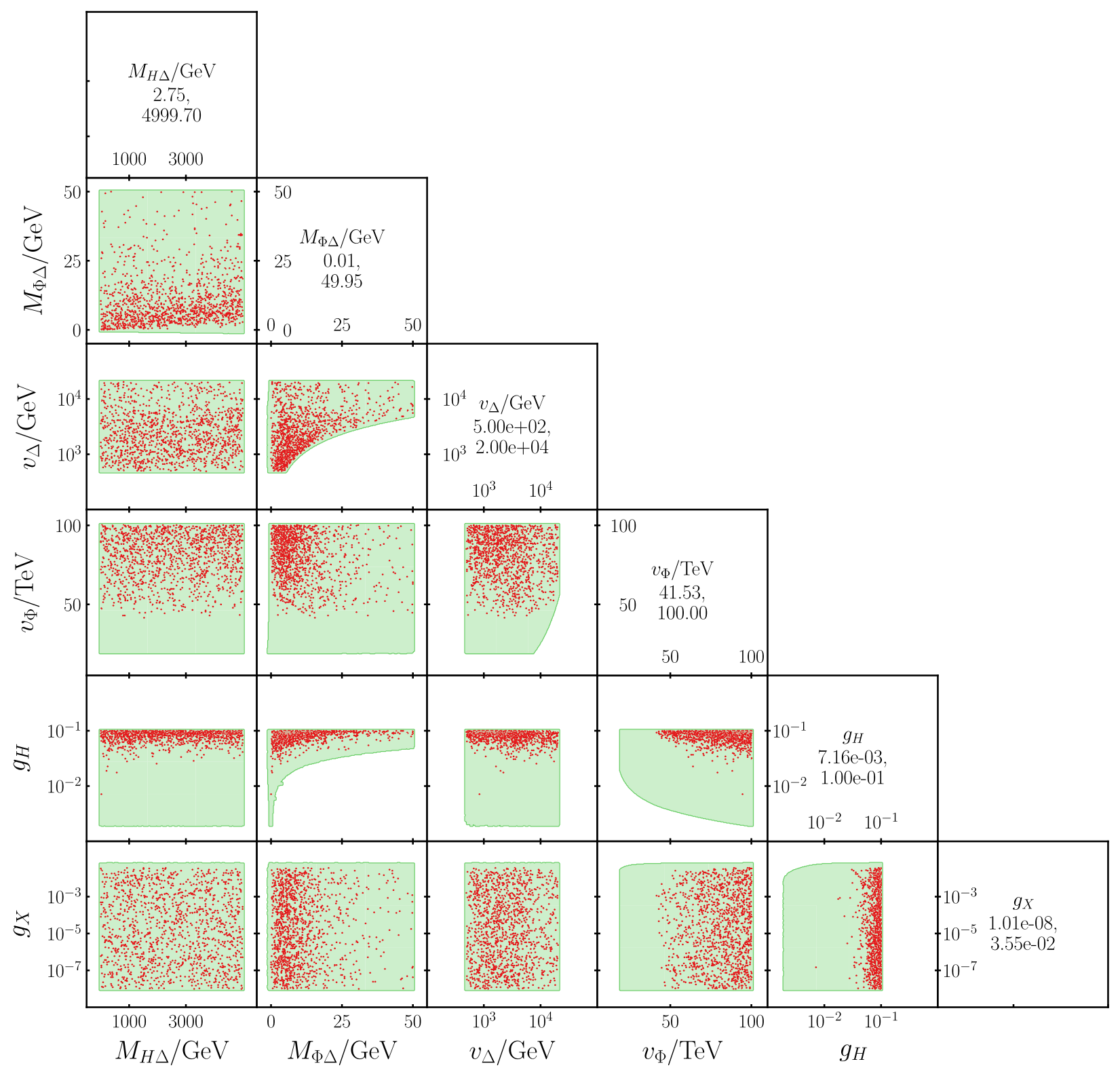

FIG. 10. A summary plot for the VEVs, $M_{\Phi \Delta}, g_{X}$, and $g_{H}$ parameter space allowed by the SGSC constraints (green region) and SGSC $+\mathrm{RD}+\mathrm{DD}$ constraints (red points) for the tripletlike DM.

(i) The allowed ranges on $\lambda_{H}$ and $\lambda_{H}^{\prime}$ remain more or less the same before and after imposing RD + DD constraints.

(ii) $\lambda_{\Phi}, \lambda_{H \Delta}$, and $\lambda_{\Phi \Delta}$ are mostly constrained by $\mathrm{RD}+$ DD constraints. To understand this effect, one can see from Eq. (A6) that there are three dominant terms that contribute to the $D D^{*} h_{j}$ couplings, $\lambda_{H \Delta} v \mathcal{O}_{11}$, $\lambda_{\Phi \Delta} v_{\Phi} \mathcal{O}_{22}$, and $\lambda_{\Delta} v_{\Delta} \mathcal{O}_{33}$ for $j=1,2,3$, respectively. Clearly, $\lambda_{H \Delta}$ and $\lambda_{\Phi \Delta}$ are restricted by the allowed Higgs coupling sizes.

(iii) Regarding to lighter mediator, in particular for $h_{2}$, the mixing $\mathcal{O}_{22}$ is strongly related to $\lambda_{\Phi}$ so that $\lambda_{\Phi}$ and $\lambda_{\Phi \Delta}$ are correlated as shown in the third row from bottom to top and second column of Fig. 9.
These two parameters are related to $h_{1}$ decay to $f \bar{f}$ and are constrained by Higgs physics and further by DD constraints.

(iv) However, $\lambda_{\Delta}$ is not constrained because either the DM annihilation or DM-nucleon elastic scattering cross section via the exchange of $h_{3}$ is suppressed by its heavy mass $m_{h_{3}}$.

(v) On the other hand, the two off-diagonal terms $\lambda_{H \Phi}$ and $\lambda_{H \Phi}^{\prime}$ are constrained mildly. This is due to the loose requirement that we set for the tripletlike DM $f_{\Delta_{p}}>2 / 3$. In fact, we checked that there can be an important contribution from the $G_{H}^{p}$ component with $f_{G_{H}^{p}}$ up to $1 / 3$. As a consequence, even though $\lambda_{H \Phi}$ and $\lambda_{H \Phi}^{\prime}$ do not appear explicitly in the coupling of 
$D D^{*} h_{i}$ given in Eq. (A6), they appear via subdominant component $G_{H}^{p}$ as seen in Eq. (A8).

Next, we project the allowed G2HDM parameter space to the two VEVs $v_{\Phi}$ and $v_{\Delta}$, the two cubic couplings $M_{H \Delta}$ and $M_{\Phi \Delta}$, and the two new gauge couplings $g_{H}$ and $g_{X}$ in Fig. 10. Again, by comparing the green regions and the red scatter points in Fig. 10, we can arrive at the following results:

(i) Strikingly, only $g_{H}$ and $v_{\Phi}$ can be further constrained by $\mathrm{RD}+\mathrm{DD}$. Interestingly, we found such an exclusion comes from the lower allowed DM mass. The allowed DM mass values range from hundreds of $\mathrm{GeV}$ to a few $\mathrm{TeV}$. This range is reflected in $g_{H}$ since the minimal value we choose for $g_{H}$ is given by Eq. (40) and depends directly on the DM mass.

(ii) The other four parameters $g_{X}, v_{\Delta}, M_{H \Delta}$, and $M_{\Phi \Delta}$ are not sensitive to the dark matter physics constraints from $\mathrm{RD}+\mathrm{DD}$.

In summary, given the setup of the parameter space in our numerical scanning, a good WIMP candidate in G2HDM is the tripletlike complex scalar with a mass $m_{D}$ in the electroweak scale, and it requires $g_{H} \gtrsim 2 \times 10^{-2}$ and $v_{\Phi} \gtrsim 30 \mathrm{TeV}$.

\section{SUMMARY AND CONCLUSION}

The G2HDM is a novel two-Higgs-doublet model with a stable DM candidate protected by an accidental discrete symmetry ( $h$ parity) without the need of imposing it by hand as in the IHDM. After $S U(2)_{H}$ symmetry breaking, the symmetry remains intact and one can find three electrically neutral potential DM candidates with odd $h$ parity: the lightest dark complex scalar $D$, heavy neutrino $\nu^{H}$, and the $S U(2)_{H}$ gauge boson $W^{\prime(p, m)}$. Though these three candidates are all interesting, we focus this paper on the most popular one, the new scalar DM $D$, which is complex and hence differ from the DM in IHDM. Unlike IHDM, the mixing between $\mathcal{Z}$-odd scalars adds a touch of complexity since DM in G2HDM not only comes from the inert doublet but may also be $S U(2)_{H}$ Goldstone-like and tripletlike. We took the dominant composition $\left(f_{j}>2 / 3\right.$ with $j=H_{2}, \Delta_{p}, G^{p}$ ) as a criteria to classify them, but the mixture between them can be simply inferred. In this paper, we have discussed these three types individually with two assumptions: that all the new non-SM heavy fermions are heavy enough to have mostly negligible contributions and that DM was thermally produced before the freeze-out temperature. We have comprehensively shown their detectability and exclusions by the current SGSC and DM constraints (mainly RD $+\mathrm{DD}$ ).

Because the DM candidate is chosen to be a complex scalar in G2HDM, the DM phenomenology becomes very rich since it has captured both features of the Higgs-portal and vector-portal DM models discussed in the literature.

For the inert doubletlike DM, we found some interesting features. First, the main difference between the inert doublet DM in IHDM and G2HDM is that in IHDM there is in general a mass splitting between the scalar $S$ and pseudoscalar $P$ components of $H_{2}^{0}$, while in G2HDM they are completely degenerate and combined into one single complex field $H_{2}^{0}=S+i P$. Recall that in IHDM there is only ZSP derivative coupling but no ZSS and ZPP derivative couplings. As long as the mass splitting between $S$ and $P$ remains larger than the exchange energy between DM and nucleons in the direct detection experiments, the interactions mediated by the $Z$ gauge boson are suppressed in IHDM. Since this splitting does not exist in G2HDM, such interactions are unsuppressed and they can bring the spin independent cross section up to $\sim 10^{-38} \mathrm{~cm}^{2}$, which is significantly above the XENON1T 95\% C.L. limit for $m_{D} \gtrsim 10 \mathrm{GeV}$ and above CRESST-III result for $m_{D} \gtrsim$ $2 \mathrm{GeV}$ (Fig. 4 right panel). On the other hand, for $m_{D} \lesssim 10 \mathrm{GeV}$, the DM is over abundant because of onshell annihilation channels in $c \bar{c}$ and $\tau^{+} \tau^{-}$(Fig. 4, left panel). Hence, we conclude that the inert doubletlike DM can be completely excluded by SGSC + RD + DD constraints.

Next, a $S U(2)_{H}$ triplet scalarlike DM was discussed. Since the composition $f_{\mathrm{H}_{2}}$ has to be tiny in order to avoid the tension with DM DD, the tripletlike DM can mostly mix with the Goldstone boson $G^{p}$. There is no $Z$-resonance region in the tripletlike DM for DM annihilation and the parameter space is more or less consistent with Higgs portal DM. However, DD is still the most stringent constraint comparing with ID and collider constraints. The allowed $\mathrm{DM}$ mass by $\mathrm{SGSC}+\mathrm{RD}+\mathrm{DD}$ is required to be heavier than $m_{D} \gtrsim 300 \mathrm{GeV}$ (Fig. 5, right panel). Despite weaker constraints coming from ID (Fig. 6, left panel) and collider searches, it might be possible to detect the heavy DM mass region by the future CTA and $100 \mathrm{TeV}$ colliders even if a DM signal is not found at direct detection experiments before hitting the neutrino floor. As shown by the blue solid boxes in the right panel of Fig. 6, the allowed tripletlike DM mass consistent with SGSC $+\mathrm{RD}+\mathrm{ID}+\mathrm{DD}$ is $\gtrsim 300 \mathrm{GeV}$.

For the last case of the Goldstone-like DM, we found that it is not possible to obtain a pure Goldstone-like DM. The nontachyonic DM condition and EWPT constraints prohibit the composition $f_{G^{p}}>0.75$ (Fig. 1), unless one would like to move to a more fine-tuned region of parameter space. Thus, there is a significant component coming from the triplet in the Goldstone-like DM. Because of the $P$-wave suppression of the $Z$ and $Z^{\prime}$ exchange in the dominated channels of $b \bar{b}$ and $W^{+} W^{-}$, the annihilation cross section happens to be smaller than for the tripletlike case and lesser points within the PLANCK relic density measurement (Fig. 7, left panel). Furthermore, XENON1T measurement excludes almost all the points with appropriate relic density, except for those with a particular value of isospin violation $\left(f_{n} / f_{p} \approx-1.86\right)$ where the sensitivity at XENON1T is reduced. Therefore, only a small region of orange boxes in the right panel of Fig. 7 with $m_{D}$ in the range of 150$600 \mathrm{GeV}$ can pass all the SGSC + DD constraints implemented in this work. For ID, the annihilation cross section at 
the present time for the Goldstone-like DM is typically smaller than the limit from Fermi gamma-ray constraints (Fig. 8, left panel). With significant ISV, only the Goldstonelike DM with a mass in the window of $150-600 \mathrm{GeV}$ can be consistent with SGSC + RD + ID + DD, as given by the blue solid boxes in the right panel of Fig. 8 .

We also presented the impact of DM constraints on the G2HDM parameter space in Figs. 9 and 10 for the tripletlike DM. In this case, we found that the following parameters $\lambda_{\Phi}, \lambda_{H \Delta}, \lambda_{\Phi \Delta}, g_{H}$, and $v_{\Phi}$ are significantly constrained by $\mathrm{DM}$ constraints, mainly $\mathrm{RD}+\mathrm{DD}$, while the four parameters $g_{X}, \lambda_{\Delta}, v_{\Delta}$, and $M_{H \Delta}$ remain more or less the same as given by the SGSC. It is interesting to note that the SGSC constraints on $g_{H}$ and $v_{\Phi}$ as studied in $[34,35]$ are now further constrained by RD + DD. We note that the lower limit of $g_{H}>7.09 \times 10^{-3}$ for $v_{\Phi}<100 \mathrm{TeV}$ is reachable by the future linear (lepton-antilepton) and $100 \mathrm{TeV}$ hadron colliders.

Before closing, we would like to make a few comments. Originally, the $S U(2)_{H}$ triplet field $\Delta_{H}$ was introduced to give mass to the charged Higgs [Eq. (20)] in [28] where the two parameters $\lambda_{H}^{\prime}$ and $\lambda_{H \Phi}^{\prime}$ were missing. With these two extra parameters included, the triplet field $\Delta_{H}$ is no longer mandatory. We note however that the triplet field $\Delta_{H}$ can give rise to a nonsingular 't Hooft-Polyakov monopole $[80,81]$ for the hidden $S U(2)_{H}$ which can play the role as DM due to its topological stability, as studied in [82]. ${ }^{7}$ Nevertheless, one can have a minimal G2HDM without the triplet field. Then the DM $D$ in this minimal model would be just mixture of the inert Higgs $H_{2}^{0 *}$ and the Goldstone field $G_{H}^{p}$. From the analysis in this work, we know that this DM scenario must be highly fine-tuned in the parameter space due to SGSC + RD + DD. A more interesting alternative DM candidate in this minimal G2HDM is the $W^{\prime(p, m)}$, which certainly deserves a separate study. Finally, whether the accidental discrete symmetry of $h$ parity, identified here in the renormalizable Lagrangian for classification of all particles in G2HDM, and the Stueckelberg mass have deeper origins remain to be explored in the future.

\section{ACKNOWLEDGMENTS}

We would like to thank Dr. Wei-Chih Huang for useful comments and discussions. T.-C. Y. would like to thank his host Professor Tri-Nang Pham and the hospitality at the center for Theoretical Physics of École Polytechnique where progress of the final phase of this work was made. This work was supported in part by the Ministry of Science and Technology (MoST) of Taiwan under Grants No. 1072119-M-001-033, No. 108-2112-M-001-018 (T.-C. Y.), No. 107-2811-M-001-027, No. 108-2811-M-001-550 (R. R.), and No. 105-2122-M-003-010-MY3 (C.-R. C.). Y.-L.S. T. was funded in part by the Chinese Academy of Sciences Taiwan Young Talent Programme under Grant No. 2018TW2JA0005.

\section{APPENDIX: FEYNMAN RULES}

Here we list the relevant couplings to the DM analysis in various processes discussed in the text. We use the conventional notations $g$ and $g^{\prime}$ to denote the SM $S U(2)_{L}$ and $U(1)_{Y}$ couplings, respectively. The $c_{W}$ and $s_{W}$ denote the cosine and sine of the Weinberg angle. The gauge couplings for $S U(2)_{H}$ and $U(1)_{X}$ are denoted by $g_{H}$ and $g_{X}$, respectively. In addition, for the scalar-scalar-gauge derivative vertices, we adopt the convention that all momenta are incoming.

\section{Four-point contact interaction}

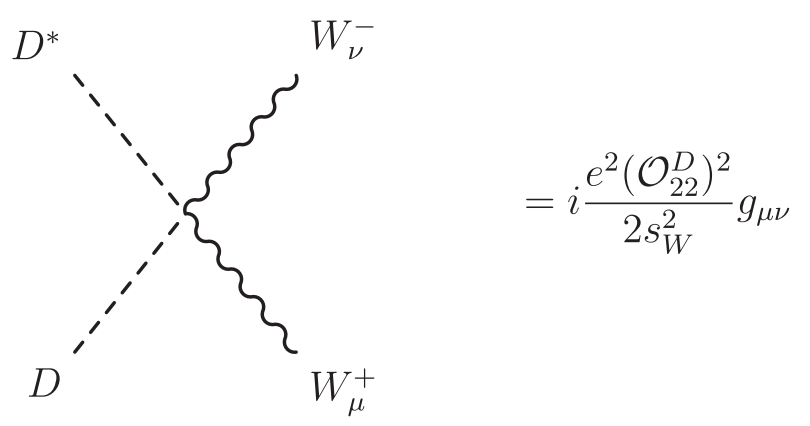

\section{Dominant couplings for inert doubletlike DM}

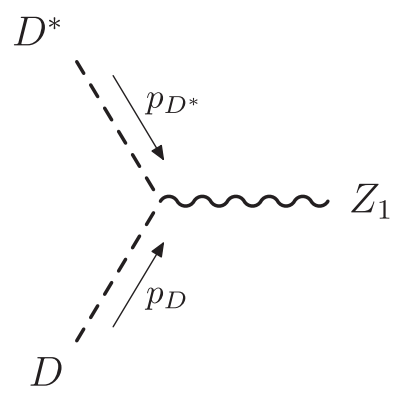

$$
\approx i\left[\frac{g c_{W}}{2}+\frac{g^{\prime} s_{W}}{2}\right]\left(\mathcal{O}_{22}^{D}\right)^{2} \mathcal{O}_{11}^{G}\left(p_{D^{*}}-p_{D}\right)_{\mu}
$$

\footnotetext{
${ }^{7}$ We thank P. Ko for bringing this reference to our attention.
} 


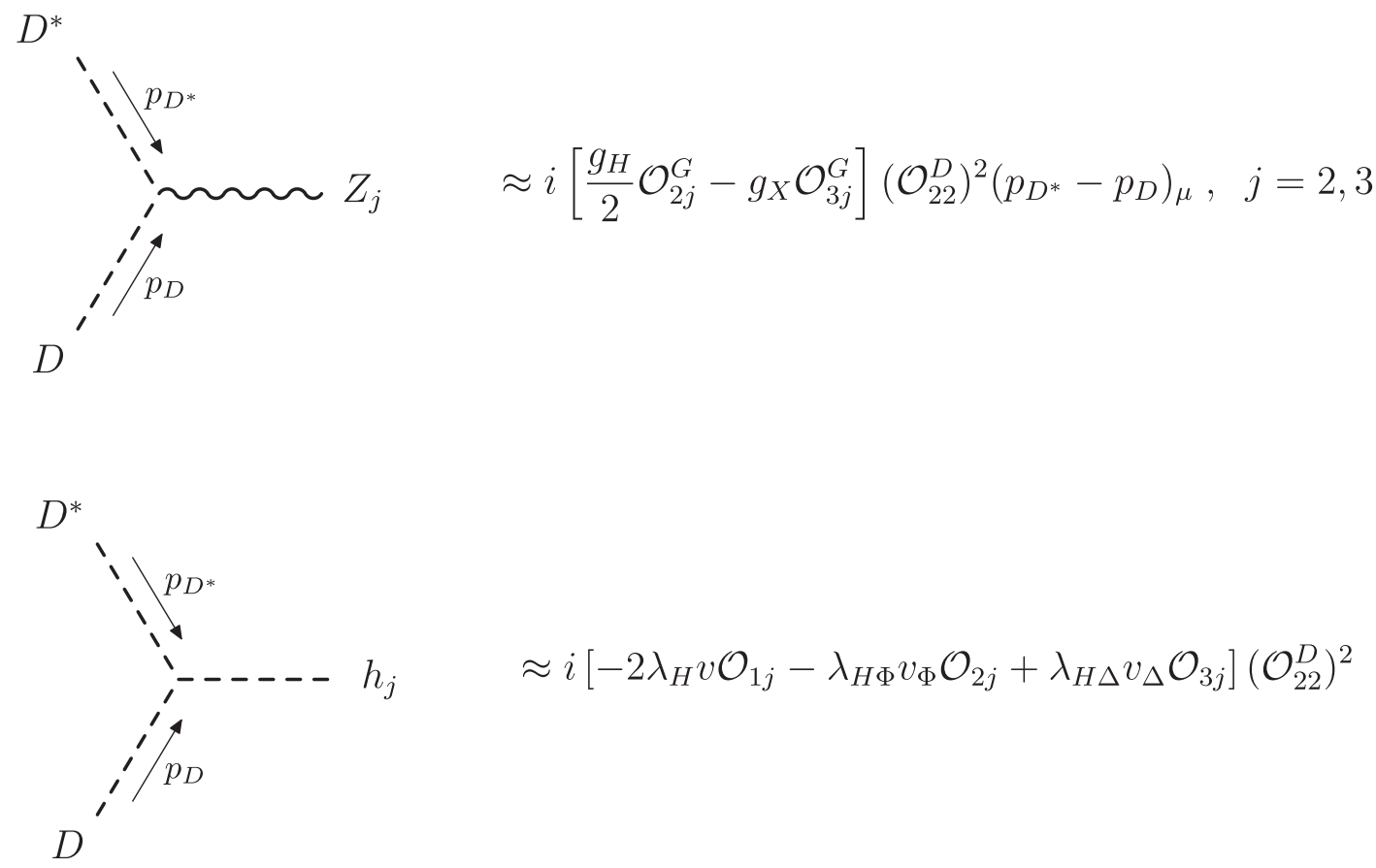

3. Dominant couplings for tripletlike DM

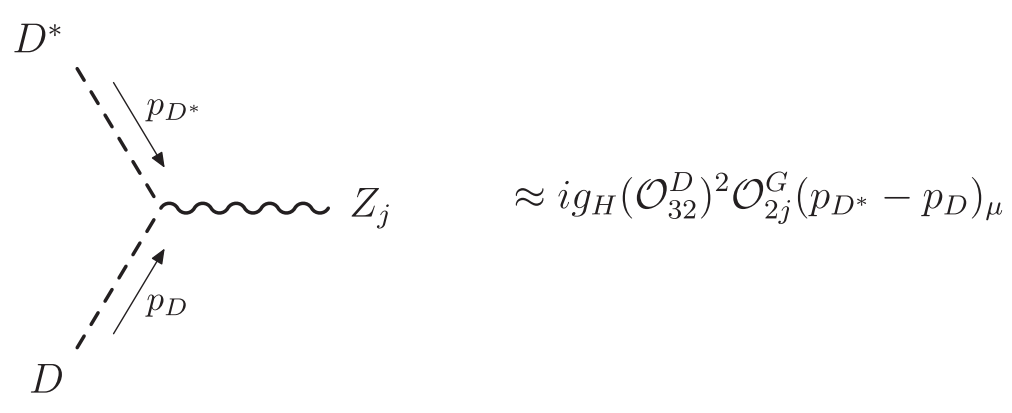

D*

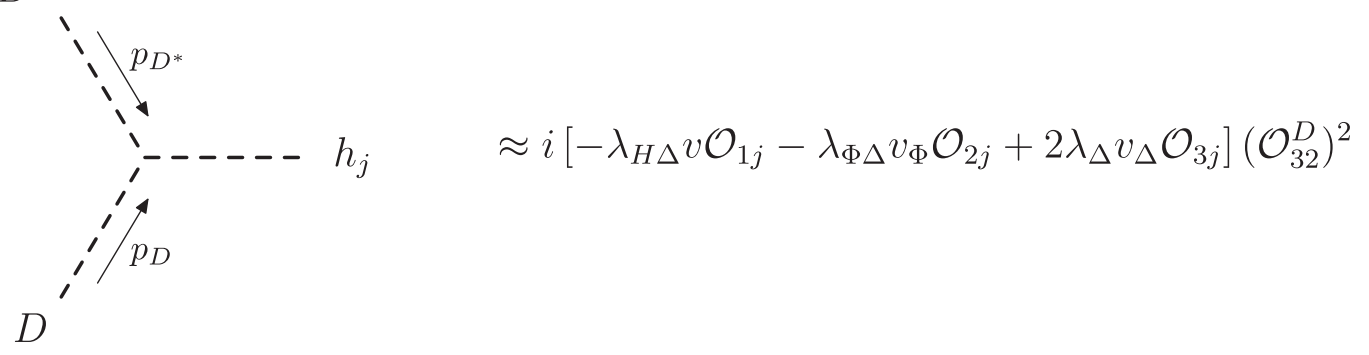




\section{Dominant couplings for Goldstone bosonlike DM}
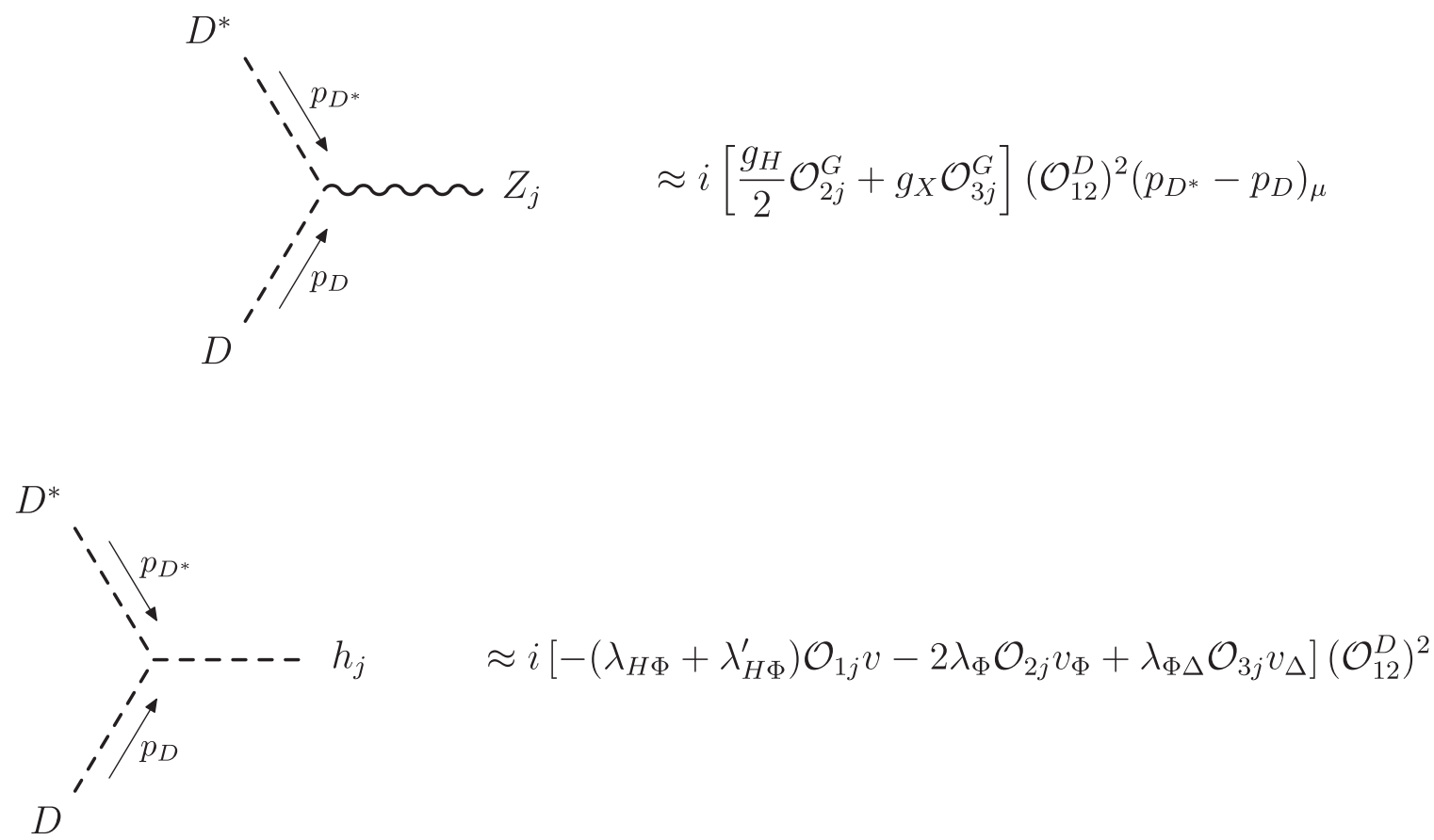

[1] G. C. Branco, P. M. Ferreira, L. Lavoura, M. N. Rebelo, M. Sher, and J. P. Silva, Phys. Rep. 516, 1 (2012).

[2] H. E. Logan, arXiv:1406.1786.

[3] N. G. Deshpande and E. Ma, Phys. Rev. D 18, 2574 (1978).

[4] L. L. Honorez, E. Nezri, J. F. Oliver, and M. H. G. Tytgat, J. Cosmol. Astropart. Phys. 02 (2007) 028.

[5] C. Arina, F. S. Ling, and M. H. G. Tytgat, J. Cosmol. Astropart. Phys. 10 (2009) 018.

[6] E. Nezri, M. H. G. Tytgat, and G. Vertongen, J. Cosmol. Astropart. Phys. 04 (2009) 014.

[7] X. Miao, S. Su, and B. Thomas, Phys. Rev. D 82, 035009 (2010).

[8] M. Gustafsson, S. Rydbeck, L. Lopez-Honorez, and E. Lundstrom, Phys. Rev. D 86, 075019 (2012).

[9] A. Arhrib, R. Benbrik, and N. Gaur, Phys. Rev. D 85, 095021 (2012).

[10] A. Arhrib, R. Benbrik, and T. C. Yuan, Eur. Phys. J. C 74, 2892 (2014).

[11] B. Swiezewska and M. Krawczyk, Phys. Rev. D 88, 035019 (2013).

[12] A. Arhrib, Y. L. S. Tsai, Q. Yuan, and T. C. Yuan, J. Cosmol. Astropart. Phys. 06 (2014) 030.

[13] A. Goudelis, B. Herrmann, and O. Stål, J. High Energy Phys. 09 (2013) 106.
[14] M. Krawczyk, D. Sokolowska, P. Swaczyna, and B. Swiezewska, J. High Energy Phys. 09 (2013) 055.

[15] M. Krawczyk, D. Sokolowska, P. Swaczyna, and B. Swiezewska, Acta Phys. Pol. B 44, 2163 (2013).

[16] A. Ilnicka, M. Krawczyk, and T. Robens, Phys. Rev. D 93, 055026 (2016).

[17] M. A. Diaz, B. Koch, and S. Urrutia-Quiroga, Adv. High Energy Phys. 2016, 8278375 (2016).

[18] K. P. Modak and D. Majumdar, Astrophys. J. Suppl. 219, 37 (2015).

[19] T. W. Kephart and T. C. Yuan, Nucl. Phys. B906, 549 (2016).

[20] F. S. Queiroz and C. E. Yaguna, J. Cosmol. Astropart. Phys. 02 (2016) 038.

[21] C. Garcia-Cely, M. Gustafsson, and A. Ibarra, J. Cosmol. Astropart. Phys. 02 (2016) 043.

[22] M. Hashemi and S. Najjari, Eur. Phys. J. C 77, 592 (2017).

[23] P. Poulose, S. Sahoo, and K. Sridhar, Phys. Lett. B 765, 300 (2017).

[24] A. Alves, D. A. Camargo, A. G. Dias, R. Longas, C. C. Nishi, and F. S. Queiroz, J. High Energy Phys. 10 (2016) 015.

[25] A. Datta, N. Ganguly, N. Khan, and S. Rakshit, Phys. Rev. D 95, 015017 (2017). 
[26] A. Belyaev, G. Cacciapaglia, I. P. Ivanov, F. Rojas-Abatte, and M. Thomas, Phys. Rev. D 97, 035011 (2018).

[27] A. Belyaev, T. R. F. P. Tomei, P. G. Mercadante, C. S. Moon, S. Moretti, S. F. Novaes, L. Panizzi, F. Rojas, and M. Thomas, Phys. Rev. D 99, 015011 (2019).

[28] W. C. Huang, Y. L. S. Tsai, and T. C. Yuan, J. High Energy Phys. 04 (2016) 019.

[29] H. Davoudiasl, R. Kitano, T. Li, and H. Murayama, Phys. Lett. B 609, 117 (2005).

[30] S. W. Ham, Y. S. Jeong, and S. K. Oh, J. Phys. G 31, 857 (2005).

[31] W. C. Huang, Y. L. S. Tsai, and T. C. Yuan, Nucl. Phys. B909, 122 (2016).

[32] W. C. Huang, H. Ishida, C. T. Lu, Y. L. S. Tsai, and T. C. Yuan, Eur. Phys. J. C 78, 613 (2018).

[33] C. R. Chen, Y. X. Lin, V. Q. Tran, and T. C. Yuan, Phys. Rev. D 99, 075027 (2019).

[34] A. Arhrib, W. C. Huang, R. Ramos, Y. L. S. Tsai, and T. C. Yuan, Phys. Rev. D 98, 095006 (2018).

[35] C. T. Huang, R. Ramos, V. Q. Tran, Y. L. S. Tsai, and T. C. Yuan, J. High Energy Phys. 09 (2019) 048.

[36] R. N. Mohapatra and G. Senjanovic, Phys. Rev. Lett. 44, 912 (1980).

[37] W. Y. Keung and G. Senjanovic, Phys. Rev. Lett. 50, 1427 (1983).

[38] P. Q. Hung, Phys. Lett. B 649, 275 (2007).

[39] P. Q. Hung, T. Le, V. Q. Tran, and T. C. Yuan, Nucl. Phys. B932, 471 (2018).

[40] P. Q. Hung, T. Le, V. Q. Tran, and T. C. Yuan, J. High Energy Phys. 12 (2015) 169.

[41] P. Ko, Y. Omura, and C. Yu, Phys. Lett. B 717, 202 (2012).

[42] M. D. Campos, D. Cogollo, M. Lindner, T. Melo, F. S. Queiroz, and W. Rodejohann, J. High Energy Phys. 08 (2017) 092.

[43] D. A. Camargo, L. Delle Rose, S. Moretti, and F. S. Queiroz, Phys. Lett. B 793, 150 (2019).

[44] D. A. Camargo, A. G. Dias, T.B. de Melo, and F. S. Queiroz, J. High Energy Phys. 04 (2019) 129.

[45] D. A. Camargo, M. D. Campos, T. B. de Melo, and F. S. Queiroz, Phys. Lett. B 795, 319 (2019).

[46] D. Cogollo, R. D. Matheus, T.B. de Melo, and F. S. Queiroz, Phys. Lett. B 797, 134813 (2019).

[47] K. Kannike, Eur. Phys. J. C 72, 2093 (2012).

[48] K. Kannike, Eur. Phys. J. C 76, 324 (2016); 78, 355(E) (2018).

[49] K. G. Klimenko, Teor. Mat. Fiz. 62, 87 (1985) [Theor. Math. Phys. 62, 58 (1985)].

[50] M. Aaboud et al. (ATLAS Collaboration), Phys. Rev. D 98, 052005 (2018).

[51] E. C. G. Stueckelberg, Helv. Phys. Acta 11, 299 (1938).

[52] H. Ruegg and M. Ruiz-Altaba, Int. J. Mod. Phys. A 19, 3265 (2004).

[53] B. Kors and P. Nath, J. High Energy Phys. 07 (2005) 069.

[54] B. Kors and P. Nath, arXiv:hep-ph/0411406.

[55] B. Kors and P. Nath, J. High Energy Phys. 12 (2004) 005.

[56] B. Kors and P. Nath, Phys. Lett. B 586, 366 (2004).
[57] D. Feldman, Z. Liu, and P. Nath, AIP Conf. Proc. 939, 50 (2007).

[58] D. Feldman, Z. Liu, and P. Nath, Phys. Rev. D 75, 115001 (2007).

[59] D. Feldman, Z. Liu, and P. Nath, J. High Energy Phys. 11 (2006) 007.

[60] S. L. Glashow and S. Weinberg, Phys. Rev. D 15, 1958 (1977).

[61] E. A. Paschos, Phys. Rev. D 15, 1966 (1977).

[62] N. Aghanim et al. (Planck Collaboration), arXiv:1807. 06209.

[63] E. Aprile et al. (XENON Collaboration), Phys. Rev. Lett. 121, 111302 (2018).

[64] J. L. Feng, J. Kumar, D. Marfatia, and D. Sanford, Phys. Lett. B 703, 124 (2011).

[65] G. Belanger, F. Boudjema, A. Goudelis, A. Pukhov, and B. Zaldivar, Comput. Phys. Commun. 231, 173 (2018).

[66] G. Belanger, F. Boudjema, A. Pukhov, and A. Semenov, Comput. Phys. Commun. 180, 747 (2009).

[67] A. Albert et al. (Fermi-LAT and DES Collaborations), Astrophys. J. 834, 110 (2017).

[68] X. Huang, Y. L. S. Tsai, and Q. Yuan, Comput. Phys. Commun. 213, 252 (2017).

[69] M. Cirelli, A poor particle physicist cookbook for dark matter indirect detection, http://www.marcocirelli.net/ PPPC4DMID.html.

[70] M. Aaboud et al. (ATLAS Collaboration), J. High Energy Phys. 01 (2018) 126.

[71] A. M. Sirunyan et al. (CMS Collaboration), Phys. Rev. D 97, 092005 (2018).

[72] M. Tanabashi et al. (Particle Data Group), Phys. Rev. D 98, 030001 (2018).

[73] http://pdg.arsip.lipi.go.id/2017/reviews/rpp2016-rev-susy2-experiment.pdf.

[74] J. Hisano, S. Matsumoto, M. M. Nojiri, and O. Saito, Phys. Rev. D 71, 063528 (2005).

[75] N. Arkani-Hamed, D. P. Finkbeiner, T. R. Slatyer, and N. Weiner, Phys. Rev. D 79, 015014 (2009).

[76] T. Hambye, F.-S. Ling, L. L. Honorez, and J. Rocher, J. High Energy Phys. 07 (2009) 090; 05 (2010) 066(E).

[77] K. Griest and M. Kamionkowski, Phys. Rev. Lett. 64, 615 (1990).

[78] A. H. Abdelhameed et al. (CRESST Collaboration), Phys. Rev. D 100, 102002 (2019).

[79] A. Morselli (CTA Consortium), Proc. Sci., ICRC2017 (2018) 921.

[80] G. 't Hooft, Nucl. Phys. B79, 276 (1974).

[81] A. M. Polyakov, Pis'ma Zh. Eksp. Teor. Fiz. 20, 430 (1974) [JETP Lett. 20, 194 (1974)].

[82] S. Baek, P. Ko, and W. I. Park, J. Cosmol. Astropart. Phys. 10 (2014) 067.

[83] J. Alwall, R. Frederix, S. Frixione, V. Hirschi, F. Maltoni, O. Mattelaer, H.-S. Shao, T. Stelzer, P. Torrielli, and M. Zaro, J. High Energy Phys. 07 (2014) 079.

[84] H. Han and S. Zheng, Nucl. Phys. B914, 248 (2017). 